\title{
On ordinals accessible by infinitary languages
}

\author{
by \\ Saharon Shelah (Jerusalem and New Brunswick, NJ), \\ Pauli Väisänen (Helsinki) and Jouko Väänänen (Helsinki)
}

\begin{abstract}
Let $\lambda$ be an infinite cardinal number. The ordinal number $\delta(\lambda)$ is the least ordinal $\gamma$ such that if $\phi$ is any sentence of $L_{\lambda+\omega}$, with a unary predicate $D$ and a binary predicate $\prec$, and $\phi$ has a model $\mathcal{M}$ with $\left\langle D^{\mathcal{M}}, \prec^{\mathcal{M}}\right\rangle$ a well-ordering of type $\geq \gamma$, then $\phi$ has a model $\mathcal{M}^{\prime}$ where $\left\langle D^{\mathcal{M}^{\prime}}, \prec^{\mathcal{M}^{\prime}}\right\rangle$ is non-well-ordered. One of the interesting properties of this number is that the Hanf number of $L_{\lambda+\omega}$ is exactly $\beth_{\delta(\lambda)}$. It was proved in [BK71] that if $\aleph_{0}<\lambda<\kappa$ are regular cardinal numbers, then there is a forcing extension, preserving cofinalities, such that in the extension $2^{\lambda}=\kappa$ and $\delta(\lambda)<\lambda^{++}$. We improve this result by proving the following: Suppose $\aleph_{0}<\lambda<\theta \leq \kappa$ are cardinal numbers such that

- $\lambda^{<\lambda}=\lambda$;

- $\operatorname{cf}(\theta) \geq \lambda^{+}$and $\mu^{\lambda}<\theta$ whenever $\mu<\theta$;

- $\kappa^{\lambda}=\kappa$.

Then there is a forcing extension preserving all cofinalities, adding no new sets of cardinality $<\lambda$, and such that in the extension $2^{\lambda}=\kappa$ and $\delta(\lambda)=\theta$.
\end{abstract}

1. Introduction. William Hanf [Han62] showed that for every logic $\mathcal{L}$ whose class of sentences is a set, there are cardinal numbers $\lambda$ such that if $\phi \in \mathcal{L}$ has a model of cardinality $\geq \lambda$, then $\phi$ has arbitrarily large models. The smallest such $\lambda$ is called the Hanf number of $\mathcal{L}$ and denoted by $h(\mathcal{L})$. It is an easy consequence of the compactness theorem that $h\left(L_{\omega \omega}\right)=\aleph_{0}$. For other infinitary languages $L_{\lambda \kappa}$ even weak versions of the compactness theorem fail unless $\lambda$ is at least weakly compact [Han64]. But there are other, more refined ways to calculate Hanf numbers.

For $\kappa>\aleph_{0}$ the number $h\left(L_{\lambda \kappa}\right)$ is quite large and hard to compute. For example, it is larger than the first weakly compact cardinal if $\mathrm{V}=\mathrm{L}$

2000 Mathematics Subject Classification: 03C75, 03E35, 03C55.

Key words and phrases: Hanf number, infinitary logic, accessible ordinal.

Research of S. Shelah partially supported by the United States-Israel Binational Science Foundation. Publication number [ShVV:812].

Research of P. Väisänen and J. Väänänen partially supported by grant 40734 of the Academy of Finland. 
([Sil71]). This is largely due to the fact that we can define well-ordering in $L_{\lambda \kappa}$ when $\kappa>\aleph_{0}$.

In contrast, well-order is not definable in the languages $L_{\lambda \omega}$ (even with extra predicates) [LE66], and methods developed first in [Mor65] give the estimates

$$
\beth_{\lambda^{+}} \leq h\left(L_{\lambda^{+} \omega}\right)<\beth_{\left(2^{\lambda}\right)^{+}} .
$$

It is known that these estimates cannot be improved in ZFC alone [BK71]. The main goal of this paper is to continue the work of [BK71] by studying what values $h\left(L_{\lambda^{+}} \omega\right)$ can possibly assume between the lower and the upper bound.

Let us call a sentence $\phi$ of some logic, in a vocabulary that includes a binary predicate $\prec$ and a unary predicate $D$, well-ordered if $\left\langle D^{\mathcal{M}}, \prec \mathcal{M}\right\rangle$ is a well-ordered structure for every model $\mathcal{M}$ of $\phi$. Let $\delta(\phi)$ be the supremum of order-types of $\left\langle D^{\mathcal{M}}, \prec^{\mathcal{M}}\right\rangle$ (if it exists) when $\mathcal{M}$ ranges over all models of $\phi$. It follows from the undefinability of well-order by extra predicates that $\delta(\phi)$ always exists if $\phi$ is a well-ordered sentence of $L_{\lambda+\omega}$. Thus we can define $\delta(\lambda)$ to be the supremum of $\delta(\phi)$ when $\phi$ ranges over all well-ordered sentences of $L_{\lambda^{+} \omega}$. The basic connection between $\delta(\lambda)$ and $h\left(L_{\lambda^{+} \omega}\right)$ was proved in [BK71]:

$$
h\left(L_{\lambda^{+} \omega}\right)=\beth_{\delta(\lambda)} .
$$

This reduced the problem of computing the Hanf number of $L_{\lambda^{+} \omega}$ to the problem of computing $\delta(\lambda)$. An old result from [Hel64] tells us that if $\lambda$ is a strong limit cardinal of cofinality $\omega$, then $\delta(\lambda)=\lambda^{+}$. In Section 3 we define a modification of the concept of tree-accessible ordinal of [BK71] and use it to make the rather easy observations:

$$
\begin{aligned}
& \delta(\lambda)<\left(2^{\lambda}\right)^{+} . \\
& \operatorname{cf}(\delta(\lambda))>\lambda . \\
& \text { If } \alpha<\delta(\lambda), \text { then } \alpha=\delta(\phi) \text { for some } \phi \in L_{\lambda^{+} \omega} .
\end{aligned}
$$

Of these, (1.3) seems to be new. For more results on $\delta(\lambda)$ see [She90, Chapter VII, §5].

It was proved in [BK71] that if $\aleph_{0}<\lambda<\kappa$ are regular cardinal numbers, then there are forcing extensions $\mathrm{V}^{P_{1}}$ and $\mathrm{V}^{P_{2}}$, preserving cofinalities, such that in both extensions $2^{\lambda}=\kappa$, while in $\mathrm{V}^{P_{1}}$ we have $\delta(\lambda)<\lambda^{++}$, and in $\mathrm{V}^{P_{2}}$ we have $\delta(\lambda)>\kappa$. We improve this result by fixing in advance the cardinal that, in the extension, will be $\delta(\lambda)$. The approach of Barwise and Kunen in [BK71] was to use Cohen forcing for $\mathrm{V}^{P_{1}}$ and a standard forcing adding dominating functions for $\mathrm{V}^{P_{2}}$. We use a more tailor made forcing. To this end we improve in Section 4 the machinery of Section 3. As a result, there is a clear idea what is the natural forcing for pushing up $\delta(\lambda)$. Section 4 
introduces this forcing and establishes its basic properties. The main result (Theorem 2) of the paper is proved in Section 5:

THEOREM 1. Suppose $\aleph_{0}<\lambda<\theta \leq \kappa$ are cardinal numbers such that

- $\lambda^{<\lambda}=\lambda$;

- $\operatorname{cf}(\theta) \geq \lambda^{+}$and $\mu^{\lambda}<\theta$ whenever $\mu<\theta$;

- $\kappa^{\lambda}=\kappa$.

Then there is a forcing extension preserving all cofinalities, adding no new sets of cardinality $<\lambda$, and such that in the extension $\delta(\lambda)=\theta$ and $2^{\lambda}=\kappa$.

The above theorem shows that $\delta(\lambda)$ need not always be of cofinality $\lambda^{+}$. This means that if we define $\delta(\gamma, \lambda)$ like $\delta(\lambda)$ but restricting ourselves to sentences of quantifier-rank $<\gamma$, then the numbers $\delta(\gamma, \lambda)$ do not have to (strictly) increase with $\gamma<\lambda^{+}$. Note that our result is for regular $\lambda$. For singular strong limit $\lambda$ of uncountable cofinality Shelah [She90, VII, §5] proved $\delta(\lambda)>2^{\lambda}$. In fact, he gets a lower bound for $\delta(\lambda)$ in terms of ranks $\|f\|_{D}$, $D$ an $\aleph_{1}$-complete filter on $\lambda$. Grossberg and Shelah [GS] get such lower bounds for a modified Hanf number, related to the infinitary (i.e. $L_{\lambda^{+} \omega}$ ) order property, even for regular $\lambda$. For another modified Hanf number, also related to the order property, they also get non-trivial upper bounds [GS86]. In view of the above theorem such bounds cannot be obtained for $\delta(\lambda)$.

2. The $\Sigma_{1}^{1}$-relations on $\mathcal{P}(\lambda)$. Throughout the paper $\lambda$ is an uncountable regular cardinal.

Definition 2.1. Suppose $\phi$ is a sentence of the infinitary language $L_{\lambda^{+} \omega}[v]$, where $v$ is a vocabulary of cardinality $\leq \lambda$ which contains a binary relation symbol $\prec$ and a unary predicate symbol $D$. We say that $\phi$ is a w.o. sentence if every model $\mathcal{M}$ of $\phi$ is such that the interpretation $\prec \mathcal{M}$ of $\prec$ is a well-ordering of the interpretation $D^{\mathcal{M}}$ of $D$.

For a w.o. sentence $\phi$, we write $\delta(\phi) \geq \alpha$ if for every $\beta<\alpha$ there is a model $\mathcal{M}$ of $\phi$ such that $\left\langle D^{\mathcal{M}}, \prec^{\mathcal{M}}\right\rangle$ has order type at least $\beta$. We write $\delta(\phi)=\alpha$ if $\delta(\phi) \geq \beta$ for every $\beta<\alpha$ and there is no model of $\phi$ such that $\left\langle D^{\mathcal{M}}, \prec^{\mathcal{M}}\right\rangle$ has order type at least $\alpha$.

Definition 2.2. A binary relation $R$ on $\mathcal{P}(\lambda)$ is called $\Sigma_{1}^{1}$ if there exists a vocabulary $v$, a model $\mathcal{M}$, and a sentence $\psi$ such that

- $\operatorname{card}(v) \leq \lambda$;

- the universe of $\mathcal{M}$ is $\lambda$;

- $\psi$ is a first order sentence of vocabulary $v$;

- $v$ contains unary predicate symbols $P_{0}, P_{1}$, and $P_{2}$;

- the vocabulary of $\mathcal{M}$ is $v \backslash\left\{P_{0}, P_{1}, P_{2}\right\}$; 
- for all $X, Y \in \mathcal{P}(\lambda), R(Y, X)$ if and only if there exists $Z \in \mathcal{P}(\lambda)$ such that

$$
\langle\mathcal{M}, X, Y, Z\rangle \models \psi
$$

where $X, Y$, and $Z$ are the interpretations of $P_{0}, P_{1}$, and $P_{2}$ respectively.

Definition 2.3. Suppose $R$ is a binary relation on $\mathcal{P}(\lambda)$. The rank $\operatorname{rk}_{R}(X)$ of $X$ in $R$, and the $\operatorname{rank} \operatorname{rk}(R)$ of $R$, are defined as usual: $\operatorname{rk}_{R}(X) \geq \alpha$ if for every $\beta<\alpha$ there exists $Y \in \mathcal{P}(\lambda)$ such that $R(Y, X)$ and $\operatorname{rk}_{R}(Y) \geq \beta$; $\operatorname{rk}_{R}(X)=\infty$ if $\operatorname{rk}_{R}(X) \geq \alpha$ for every $\alpha \in \mathrm{On} ; \operatorname{rk}(R)=\infty$ if for every $\alpha \in$ On there is $X \in \mathcal{P}(\lambda)$ such that $\operatorname{rk}_{R}(X) \geq \alpha$, otherwise $\operatorname{rk}(R)=$ $\sup \left\{\operatorname{rk}_{R}(X) \mid X \in \mathcal{P}(\lambda)\right\}$.

Lemma 2.4. For every $\alpha<\delta(\lambda)$ there is a $\Sigma_{1}^{1}$-relation $R$ on $\mathcal{P}(\lambda)$ such that $\operatorname{rk}(R) \neq \infty$ and $\operatorname{rk}(R) \geq \alpha$.

Proof. Suppose $\alpha<\delta(\lambda)$ and $\phi$ is a w.o. sentence in $L_{\lambda^{+} \omega}[v]$ such that $\delta(\phi) \geq \alpha+1$. We define a $\Sigma_{1}^{1}$-relation $R_{\phi}$ having the desired properties.

Remember that $D$ and $\prec$ are symbols in $v$ as in Definition 2.1. Without loss of generality we may assume that $v$ has Skolem functions and hence the class of models of $\phi$ is closed under submodels and unions of increasing chains of models. Moreover, we may assume that $\alpha \geq \lambda$, otherwise it is easy to define a suitable $R_{\phi}$.

Define $A_{n}$ to be $\{(\omega \cdot i)+l \mid i<\lambda$ and $l<n\}$ for every $n<\omega$. Using a suitable coding, say $\pi$, we may assume that every $X \subseteq \lambda$ codes a pair $\left\langle\mathcal{M}_{X}, c_{X}\right\rangle$ of a $v$-model $\mathcal{M}_{X}$ and an element $c_{X} \in \mathcal{M}_{X}$ such that the universe of $\mathcal{M}_{X}$ is a subset of $A_{n}$ for some $n<\omega$. Moreover, we may assume that $\pi$ is such that

- if $m \leq n<\omega, X \subseteq A_{m}$, and $Y \subseteq A_{n}$, then $X=Y \cap A_{m}$ implies $\mathcal{M}_{X} \subseteq \mathcal{M}_{Y}$

- for every $\langle\mathcal{N}, d\rangle$, if $\mathcal{N}$ has universe $A_{n}$ for some $n<\omega$, then there is $X \subseteq A_{n}$ such that $\mathcal{M}_{X}=\mathcal{N}$ and $c_{X}=d$;

- for all $v$-models $\mathcal{N}_{1} \subseteq \mathcal{N}_{2}$ and $n<\omega$, if $\mathcal{N}_{1}$ has universe $A_{n}$ and $\mathcal{N}_{2}$ has universe $A_{n+1}$, then for those $X_{1} \subseteq A_{n}$ and $X_{2} \subseteq A_{n+1}$ coding $\left\langle\mathcal{N}_{1}, d_{1}\right\rangle$ and $\left\langle\mathcal{N}_{2}, d_{2}\right\rangle$ for some $d_{1}$ and $d_{2}$ respectively, we have $X_{1}=X_{2} \cap A_{n}$.

For all $X, Y \subseteq \lambda$ define $R_{\phi}(Y, X)$ if there exists $n<\omega$ such that

- $X \subseteq A_{n}$ and $Y \subseteq A_{n+1}$;

- $X=Y \cap A_{n}$ (which implies $\mathcal{M}_{X} \subseteq \mathcal{M}_{Y}$ );

- $\mathcal{M}_{X} \models \phi$ and $\mathcal{M}_{Y} \models \phi$;

- $c_{X} \in D^{\mathcal{M}_{X}}, c_{Y} \in D^{\mathcal{M}_{Y}}$, and $c_{Y} \prec \mathcal{M}_{Y} c_{X}$.

We may assume that $\phi$ is coded as a subset of $\lambda$. Hence there exists a first order sentence $\psi$ and a parameter $U \subseteq \lambda$ such that for all $X \subseteq \lambda$, $\langle\lambda, \pi, X, U\rangle \models \psi$ if and only if $\mathcal{M}_{X} \models \phi$. Hence $R_{\phi}$ is a $\Sigma_{1}^{1}$-relation on $\mathcal{P}(\lambda)$. 
Necessarily we have $\operatorname{rk}\left(R_{\phi}\right) \neq \infty$. Namely, if $\operatorname{rk}\left(R_{\phi}\right)=\infty$ then there exists $\left\langle X_{n} \mid n<\omega\right\rangle$ such that $R_{\phi}\left(X_{n+1}, X_{n}\right)$ for every $n<\omega$. By the definition $\left\langle\mathcal{M}_{X_{n}} \mid n<\omega\right\rangle$ is an increasing chain of models of $\phi$ and $c_{X_{n+1}} \prec \mathcal{M}_{X_{n+1}} c_{X_{n}}$. By the assumption on Skolem functions, $\mathcal{N}=\bigcup_{n<\omega} \mathcal{M}_{X_{n}}$ is a model of $\phi$ such that $\left\langle D^{\mathcal{N}}, \prec^{\mathcal{N}}\right\rangle$ is not well-ordered. This contradicts the choice of $\phi$.

Define $\Gamma(\alpha)$ to be the set of all (strictly) descending sequences of ordinals below $\alpha$. To prove $\operatorname{rk}\left(R_{\phi}\right) \geq \alpha$, it suffices to find $X_{\eta}$, for every $\eta \in \Gamma(\alpha)$, so that $R_{\phi}\left(X_{\nu}, X_{\eta}\right)$ whenever $\nu=\eta \frown\langle\xi\rangle \in \Gamma(\alpha)$ for some $\xi \in \alpha$.

Let $\mathcal{N}$ be a model of $\phi$ such that the order type of $\left\langle D^{\mathcal{N}}, \prec \mathcal{N}\right\rangle$ is at least $\alpha$. By induction on $\operatorname{lh}(\eta), \eta \in \Gamma(\alpha)$, define sets $X_{\eta}$, functions $f_{\eta}$, and models $\mathcal{N}_{\eta}$ as follows. Set $X_{\emptyset}=f_{\emptyset}=\mathcal{N}_{\emptyset}=\emptyset$. Suppose $\eta, \nu \in \Gamma(\alpha), \nu=\eta \frown\langle\xi\rangle$, $\operatorname{lh}(\eta)=n$, and $X_{\eta}, f_{\eta}, \mathcal{N}_{\eta}$ are already chosen. Moreover, suppose that if $n \neq 0$, then

- $\mathcal{N}_{\eta}$ is a submodel of $\mathcal{N}\left(\mathcal{N}_{\eta} \models \phi\right.$ by the assumption on Skolem functions);

- $\operatorname{card}\left(\mathcal{N}_{\eta}\right)=\lambda($ remember $\operatorname{card}(v) \leq \lambda)$;

- $X_{\eta} \subseteq A_{n}$;

- $\mathcal{M}_{X_{\eta}}$ has universe $A_{n}$;

- $f_{\eta}$ is an isomorphism between $\mathcal{N}_{\eta}$ and $\mathcal{M}_{X_{\eta}}$;

- the $\prec^{\mathcal{N}}$-order type of $\left\{x \in D^{\mathcal{N}} \mid x \prec \mathcal{N} c_{X_{\eta}}\right\}$ is $\eta(n-1)$.

By the choice of $\pi$, we can choose $X_{\nu} \subseteq A_{n+1}, \mathcal{N}_{\nu} \subseteq \mathcal{N}$, and an isomorphism $f_{\nu}: \mathcal{N}_{\nu} \cong \mathcal{M}_{X_{\nu}}$ such that the conditions above become satisfied when $\eta$ and $n$ are replaced with $\nu$ and $n+1$ respectively. Moreover, we can guarantee that $\mathcal{N}_{\eta} \subseteq \mathcal{N}_{\nu}$ and $f_{\eta} \subseteq f_{\nu}$ (which implies $\mathcal{M}_{X_{\eta}} \subseteq \mathcal{M}_{X_{\nu}}$, and hence $X_{\nu}=$ $\left.X_{\eta} \cap A_{n}\right)$.

Lemma 2.5. For all $\Sigma_{1}^{1}$-relations $R$ on $\mathcal{P}(\lambda)$, either $\operatorname{rk}(R)=\infty$ or there exists a w.o. sentence $\phi_{R}$ in $L_{\lambda^{+}}{ }_{\omega}$ such that $\delta\left(\phi_{R}\right)=\operatorname{rk}(R)$.

Proof. Suppose $R$ is a $\Sigma_{1}^{1}$-relation on $\mathcal{P}(\lambda)$ such that $\operatorname{rk}(R)=\alpha+1$ and $v, \psi, \mathcal{M}$ are objects witnessing $R$ to be a $\Sigma_{1}^{1}$-relation (obviously the possible ranks of $\Sigma_{1}^{1}$-relations are closed under successor ordinals). We define a w.o. sentence $\phi_{R}$ in $L_{\lambda^{+} \omega}$ such that $\delta\left(\phi_{R}\right) \geq \alpha+1$.

As above, $\Gamma(\alpha)$ denotes the set of all descending sequences of ordinals below $\alpha$. By induction on $\eta \in \Gamma(\alpha)$ choose a subset $X_{\eta}$ of $\lambda$ such that

- $\operatorname{rk}_{R}\left(X_{\eta}\right) \geq \alpha$ if $\eta=\emptyset$;

- $\operatorname{rk}_{R}\left(X_{\eta}\right) \geq \eta(n)$ if $\eta \neq \emptyset$, where $n=\operatorname{lh}(\eta)-1$.

Moreover, we can guarantee that, if $\nu=\eta \frown\langle\xi\rangle \in \Gamma(\alpha)$, then

$$
\left\langle\mathcal{M}, X_{\eta}, X_{\nu}, Z_{\nu}\right\rangle \models \psi \quad \text { for some fixed } Z_{\nu} \subseteq \lambda
$$

(which means $R\left(X_{\nu}, X_{\eta}\right)$ by the choice of $\mathcal{M}$ and $\psi$ ). 
Now define a vocabulary $v^{+}$and a $v^{+}$-model $\mathcal{N}$ as follows. First of all $v^{+}$is $v \cup\{D, \prec\} \cup\left\{Q_{0}, \ldots, Q_{5}\right\} \cup\left\{c_{i} \mid i<\lambda\right\}$ (we may assume that the new symbols are not in $v$ ). The universe of $\mathcal{N}$ is $\mathcal{M} \cup(\alpha+1) \cup \Gamma(\alpha)$ (we may assume $\alpha \geq \lambda$ and $\mathcal{M} \cap((\alpha+1) \cup \Gamma(\alpha))=\emptyset)$. For all $S \in v$, set $S^{\mathcal{N}}=S^{\mathcal{M}}$. Moreover, define the interpretations of the rest of the symbols as follows:

- $D^{\mathcal{N}}=\alpha+1$;

- $\prec^{\mathcal{N}}=\in \uparrow(\alpha+1)$;

- $Q_{0}^{\mathcal{N}}=\mathcal{M}$

- $Q_{1}^{\mathcal{N}}=\lambda$;

- $c_{i}^{\mathcal{N}}=i$ for every $i<\lambda$ (remember that $\mathcal{M}$ has universe $\lambda$ );

- $Q_{2}^{\mathcal{N}}=\left\{\eta \mid \eta\right.$ is a finite $\prec^{\mathcal{N}}$-descending sequence of elements of $\left.D^{\mathcal{N}}\right\}$;

- $Q_{3}^{\mathcal{N}}=\left\{\langle\eta, \operatorname{lh}(\eta), l, \eta(l)\rangle \mid \eta \in Q_{2}^{\mathcal{N}}, l<\operatorname{lh}(\eta)\right\}$

- $Q_{4}^{\mathcal{N}}=\left\{\langle\eta, i\rangle \mid \eta \in Q_{2}^{\mathcal{N}}\right.$ and $\left.i \in X_{\eta}\right\}$;

- $Q_{5}^{\mathcal{N}}=\left\{\langle\eta, i\rangle \mid \eta \in Q_{2}^{\mathcal{N}} \backslash\{\emptyset\}\right.$ and $\left.i \in Z_{\eta}\right\}$.

Then define $\phi_{R}$ to be a conjunction of all relevant $L_{\lambda^{+}} \omega^{\text {-sentences holding }}$ in $\mathcal{N}$, e.g., $\phi_{R}$ contains a conjunction of the first order theory of $\mathcal{N}$ and the following:

if $\eta, \nu \in Q_{2}, " \nu=\eta \frown\langle\xi\rangle$ " (use $Q_{3}$ to denote this), then the model $\left\langle Q_{0}, Q_{3} \cap(\{\eta\} \times \lambda), Q_{3} \cap(\{\nu\} \times \lambda), Q_{4} \cap(\{\nu\} \times \lambda)\right\rangle$ satisfies $\psi$.

By the choice of $D^{\mathcal{N}}$ and $\prec \mathcal{N}, \delta\left(\phi_{R}\right) \geq \alpha+1$. It remains to prove that $\delta\left(\phi_{R}\right) \leq \alpha+1$. Suppose, contrary to the claim, that $\mathcal{N}$ is a model of $\phi_{R}$ such that $\left\langle D^{\mathcal{N}}, \prec^{\mathcal{N}}\right\rangle$ has order type $\geq \alpha+2$. We show that $\operatorname{rk}(R) \geq \alpha+2$, a contradiction.

Let $\left\langle d_{l} \mid l<n^{*}\right\rangle$ be a sequence of elements of $D^{\mathcal{N}}$ such that $n^{*} \geq 2$ and $d_{n+1} \prec^{\mathcal{N}} d_{n}$ for every $n<n^{*}-1$. Let $\eta_{n}$ be a shorthand for $\left\langle\bar{d}_{l}\right|$ $l<n\rangle$ for every $n \leq n^{*}$. By the choice of $\phi_{R}, \eta_{n}$ is in $Q_{2}^{\mathcal{N}}$. Define $X_{n}$, $n \leq n^{*}$, to be $\left\{i<\lambda \mid\left\langle\eta_{n}, c_{i}^{\mathcal{N}}\right\rangle \in Q_{4}^{\mathcal{N}}\right\}$, and if $n<n^{*}-1$, let $Z_{n+1}$ be $\left\{i<\lambda \mid\left\langle\eta_{n}, c_{i}^{\mathcal{N}}\right\rangle \in Q_{5}^{\mathcal{N}}\right\}$. It follows (provided that $\phi_{R}$ is correctly chosen) that $\left\langle\mathcal{M}, X_{\eta_{n}}, X_{\eta_{n+1}}, Z_{\eta_{n+1}}\right\rangle \models \psi$ for every $n<n^{*}-1$, where $\mathcal{M}=Q_{0}^{\mathcal{N}}$. Hence $R\left(X_{\eta_{n+1}}, X_{\eta_{n}}\right)$ for every $n<n^{*}-1$.

The $\Sigma_{1}^{1}$-relations provide very easy proofs for the following facts.

Corollary 2.6.

(a) $\delta(\lambda)<\left(2^{\lambda}\right)^{+}$

(b) $\operatorname{cf}(\delta(\lambda))>\lambda$;

(c) the set $\left\{\delta(\phi) \mid \phi\right.$ is a w.o. sentence in $\left.L_{\lambda+\omega}\right\}$ is an initial segment of ordinals below $\left(2^{\lambda}\right)^{+}$.

Proof. (a) follows from the definition of a $\Sigma_{1}^{1}$-relation and Lemma 2.4.

(b) follows from the fact that the ranks of $\Sigma_{1}^{1}$-relations are closed under unions of length $\leq \lambda$, i.e., if $\left\langle R_{i} \mid i<\lambda\right\rangle$ are $\Sigma_{1}^{1}$-relations on $\mathcal{P}(\lambda)$, then there exists a $\Sigma_{1}^{1}$-relation $R$ on $\mathcal{P}(\lambda)$ such that $\operatorname{rk}(R) \geq \bigcup_{i<\lambda} \operatorname{rk}\left(R_{i}\right)$. 
(c) follows from Lemma 2.5 and the fact that $\left\{\operatorname{rk}_{R}(X) \mid X \subseteq \lambda\right\}$ is an initial segment of the ordinals for every relation $R$ on $\mathcal{P}(\lambda)$.

3. The $\omega$-sequences of $\lambda$-trees. In this section we define auxiliary devices, $\omega$-sequences of $\lambda$-trees, needed in the proof of the consistency result presented in the next section. Under the assumption $\lambda^{<\lambda}=\lambda$, this notion provides representations for all the $\Sigma_{1}^{1}$-relations with different ranks.

Definition 3.1. A sequence $\mathbf{T}=\left\langle T_{n} \mid n<\omega\right\rangle$ is called an $\omega$-sequence of $\lambda$-trees if the following conditions are satisfied:

- $T_{0}=\{\emptyset\}$

- for every non-zero $n<\omega, T_{n}$ consists of sequences $\mathbf{t}=\left\langle t_{l} \mid l<n\right\rangle$ such that for some $\gamma \in \lambda$, each $t_{l}$ is a function from $\gamma$ into $\lambda$ (for $\mathbf{t} \in T_{n}, \operatorname{ht}(\mathbf{t})$ denotes 0 if $\mathbf{t}=\emptyset$, and otherwise, the ordinal $\gamma$ which is the domain of every function in $\mathbf{t}$ );

- for every $m<n<\omega, T_{m}=\left\{\mathbf{t}|m| \mathbf{t} \in T_{n}\right\}$ ( $T_{m}$ is a "projection" of $\left.T_{n}\right)$;

- for every $n<\omega, \unlhd$ is the following tree order on $T_{n}$ : for all $\mathbf{t}=\left\langle t_{l}\right|$ $l<n\rangle$ and $\mathbf{s}=\left\langle s_{l} \mid l<n\right\rangle$ in $T_{n}, \mathbf{t} \unlhd \mathbf{s}$ if $t_{l} \subseteq s_{l}$ for all $l<n$;

- for every $n<\omega, T_{n}$ is closed under $\unlhd$-initial segments.

For nodes $\mathbf{t}$ in $\mathbf{T}$ and $\beta<\operatorname{ht}(\mathbf{t}), \mathbf{t} \downarrow \beta$ denotes the $\unlhd$-initial segment $\left\langle t_{l} \uparrow \beta\right|$ $l<\operatorname{lh}(\mathbf{t})\rangle$ of $\mathbf{t}$.

To simplify our notation in the next section, we establish the following notation.

Definition 3.2. Suppose $\mathbf{T}$ is an $\omega$-sequence of $\lambda$-trees. The height of $\mathbf{T}$, denoted by $\mathrm{ht}(\mathbf{T})$, is the ordinal $\delta$ such that for every non-zero $n<\omega, \delta$ is the smallest ordinal satisfying ht $(\mathbf{t})<\delta$ for all $\mathbf{t} \in T_{n}$ (such a common $\delta$ exists because $T_{m}$ is a "projection" of $T_{n}$ for all $\left.0<m<n<\omega\right)$. A branch through $T_{n}$ is a sequence $\mathbf{b}$ such that

- $\mathbf{b}=\emptyset$ if $n=0$

- if $n>0$ and $\delta=\operatorname{ht}\left(T_{n}\right)$ is a limit ordinal, then $\mathbf{b}=\left\langle b_{l} \mid l<n\right\rangle$ for some functions $b_{l}$ satisfying $\operatorname{dom}\left(b_{l}\right)=\delta$ and $\mathbf{b} \downarrow \gamma \in T_{n}$ for every $\gamma<\delta$

- if $\operatorname{ht}\left(T_{n}\right)$ is a successor ordinal, say $\delta+1$, then $\mathbf{b} \in\left\{\mathbf{t} \in T_{n} \mid \operatorname{ht}(\mathbf{t})\right.$ $=\delta\}$.

The set of all branches through $T_{n}$ is denoted by $\operatorname{Br}\left(T_{n}\right)$.

Definition 3.3. Suppose $\mathbf{T}$ is an $\omega$-sequence of $\lambda$-trees. We define a rank function $\mathrm{rk}_{\mathbf{T}}$ by induction on ordinals as follows: For all $n<\omega$ and $\mathbf{b} \in \operatorname{Br}\left(T_{n}\right)$, 
$\operatorname{rk}_{\mathbf{T}}(\mathbf{b}) \geq \alpha$ if for every $\beta<\alpha$, there exists $\mathbf{c} \in \operatorname{Br}\left(T_{n+1}\right)$ such that $\mathbf{b} \subseteq \mathbf{c}$ and $\operatorname{rk}_{\mathbf{T}}(\mathbf{c}) \geq \beta$.

Moreover we define

- $\operatorname{rk}_{\mathbf{T}}(\mathbf{b})=\infty$ if $\operatorname{rk}_{\mathbf{T}}(\mathbf{b}) \geq \alpha$ for all $\alpha \in$ On, and

- $\operatorname{rk}_{\mathbf{T}}(\mathbf{b})=\alpha$ if $\operatorname{rk}_{\mathbf{T}}(\mathbf{b}) \geq \alpha$ and $\operatorname{rk}_{\mathbf{T}}(\mathbf{b}) \geq \alpha+1$.

We denote by $\operatorname{rk}(\mathbf{T})$ the rank of the trivial branch $\emptyset$ through $T_{0}$ (by Definition 3.1, $\operatorname{rk}(\mathbf{T})$ equals $\infty$ or the supremum of the ranks of the branches through $T_{1}$ ).

Lemma 3.4. Suppose $\lambda$ is an uncountable cardinal such that $\lambda^{<\lambda}=\lambda$. Then there exists an $\omega$-sequence $\mathbf{T}$ of $\lambda$-trees such that $\operatorname{rk}(\mathbf{T}) \geq \alpha$ and $\operatorname{rk}(\mathbf{T}) \neq \infty$ if and only if there exists a $\Sigma_{1}^{1}$-relation $R$ on $\mathcal{P}(\lambda)$ such that $\operatorname{rk}(R) \geq \alpha$ and $\operatorname{rk}(R) \neq \infty$.

Proof. Suppose $\mathbf{T}$ is an $\omega$-sequence of $\lambda$-trees. We define a binary relation $R_{\mathbf{T}}$ on $\mathcal{P}(\lambda)$ as follows. Let $\pi$ be a coding such that every $X \subseteq \lambda$ codes a finite sequence $\mathbf{b}^{X}$ of functions in ${ }^{\lambda} \lambda$. Moreover, suppose that $\mathbf{b}^{\emptyset}=\emptyset$ and every finite sequence of functions in ${ }^{\lambda} \lambda$ is coded by some $X \subseteq \lambda$. For all $X, Y \in \mathcal{P}(\lambda)$, define $R_{\mathbf{T}}(Y, X)$ if

- there is $n<\omega$ such that $\ln \left(\mathbf{b}^{X}\right)=n$ and $\ln \left(\mathbf{b}^{Y}\right)=n+1$;

- $\mathbf{b}^{X} \subseteq \mathbf{b}^{Y}$ and $\mathbf{b}^{Y}$ is a branch through $T_{n+1}$ (in this case $\mathbf{b}^{X}$ is a branch through $T_{n}$ ).

Since $\lambda^{<\lambda}=\lambda$, we have $\operatorname{card}(H(\lambda))=\lambda$ (the set of all elements whose transitive closure has cardinality $\leq \lambda)$. Furthermore $T_{n} \subseteq H(\lambda)$ for every $n<\omega$. It follows that there exists a first order sentence $\psi$ of vocabulary $\left\{\in, Q_{0}, Q_{1}, P_{0}, P_{1}, P_{2}\right\}$ such that for all $X, Y \subseteq \lambda, R_{\mathbf{T}}(Y, X)$ if and only if $\langle H(\lambda), \in, \pi, \mathbf{T}, X, Y, Z\rangle \models \psi$ for some $Z \subseteq \lambda$. Therefore $R_{\mathbf{T}}$ is a $\Sigma_{1}^{1}$-relation (since $\lambda^{<\lambda}=\lambda$, choose $\mathcal{M}$ having universe $\lambda$ such that it is isomorphic to $\langle H(\lambda), \in, \pi, \mathbf{T}\rangle)$. Clearly $\operatorname{rk}\left(R_{\mathbf{T}}\right) \geq \alpha$ if $\operatorname{rk}(\mathbf{T}) \geq \alpha$. Furthermore, if $\operatorname{rk}\left(R_{\mathbf{T}}\right)=$ $\infty$ then there exists an " $R_{\mathbf{T}^{-}}$descending" sequence $\left\langle X_{n} \mid n<\omega\right\rangle$ of subsets of $\lambda$ such that $\mathbf{b}^{X_{n}} \in \operatorname{Br}\left(T_{n}\right)$ and $\mathbf{b}^{X_{n}} \subseteq \mathbf{b}^{X_{n+1}}$ for every $n<\omega$. Thus $\operatorname{rk}\left(R_{\mathbf{T}}\right)=\infty$ implies $\operatorname{rk}(\mathbf{T})=\infty$.

Suppose $R$ is a $\Sigma_{1}^{1}$-relation on $\mathcal{P}(\lambda)$ and the objects $v, \mathcal{M}$, and $\psi$ witness this fact. Firstly define the following sets for every function $f$ having domain in $\lambda \cup\{\lambda\}$ and range $\lambda$ :

$$
\begin{aligned}
X_{f} & =\{f(\omega \cdot \xi+3 \cdot k) \mid \xi<\lambda \text { and } k<\omega\}, \\
Z_{f} & =\{f(\omega \cdot \xi+3 \cdot k+1) \mid \xi<\lambda \text { and } k<\omega\}, \\
C_{f} & =\{f(\omega \cdot \xi+3 \cdot k+2) \mid \xi<\lambda \text { and } k<\omega\} .
\end{aligned}
$$

Then define an $\omega$-sequence $\mathbf{T}^{R}$ of $\lambda$-trees as follows. Set $T_{0}^{R}=\{\emptyset\}$ and for every $n<\omega$, define $\mathbf{t}=\left\langle t_{l} \mid l<n+1\right\rangle \in T_{n+1}^{R}$ if 
- $\mathbf{t}\left\lceil n \in T_{n}^{R}\right.$

- if $n=0, t_{0}$ is a function from $\delta$ into $\lambda$ for some $\delta \in \lambda$;

- if $n \neq 0, t_{n}$ is a function from $\delta$ into $\lambda$ where $\delta=\operatorname{dom}\left(f_{n-1}\right)$;

- if $n \neq 0$ and $\delta$ is a limit ordinal, $f(\alpha+(3 \cdot k)+2) \geq \alpha+3 \cdot k$ for all $k<\omega$ and $\alpha<\delta$ of the form $\omega \cdot \xi$ for some $\xi<\lambda$, and moreover, $C_{t_{n}}$ is closed under supremums;

- if $n \neq 0$, then for every $\gamma \in C_{t_{n}}$, there exist $X, Y, Z \subseteq \lambda$ such that $\langle\mathcal{M}, X, Y, Z\rangle \models \psi$ and $\left\langle M \uparrow \gamma, X_{t_{n-1}}, X_{t_{n}}, Z_{t_{n}}\right\rangle$ is an elementary submodel of $\langle\mathcal{M}, X, Y, Z\rangle$ (recall that $\mathcal{M}$ must have universe $\lambda$ ).

It follows that $T_{n}$ is closed under $\unlhd$-initial segments for every $n<\omega$. To guarantee that $T_{m}$ is a "projection" of $T_{n}$ if $m<n<\omega$, we may assume that $\operatorname{rk}(R)$ is a limit ordinal and demand that for every $\mathbf{t} \in T_{n+1}^{R}$, $\left\langle\mathcal{M}, X_{t_{n}}, Y, Z\right\rangle \models \psi$ for some $Y, Z \subseteq \lambda$. Hence $\mathbf{T}^{R}$ is an $\omega$-sequence of $\lambda$-trees having height $\lambda$. Moreover, assuming that $\operatorname{rk}(R) \geq \alpha \geq \lambda, \operatorname{rk}\left(\mathbf{T}^{R}\right) \geq \alpha$.

Suppose $\operatorname{rk}\left(\mathbf{T}^{R}\right)=\infty$. Then there exist functions $\left\langle b_{l} \mid l<\omega\right\rangle$ from $\lambda$ into $\lambda$ such that $\left\langle b_{l} \mid l<n\right\rangle \in \operatorname{Br}\left(T_{n}^{R}\right)$ for every $n<\omega$. We claim that $R\left(X_{b_{l+1}}, X_{b_{l}}\right)$ for every $l<\omega$, and hence $\operatorname{rk}(R)=\infty$. Fix $l<\omega$. We show that $\left\langle\mathcal{M}, X_{b_{l}}, X_{b_{l+1}}, Z_{b_{l+1}}\right\rangle \models \psi$. Suppose, contrary to this claim, that the model satisfies $\neg \psi$. Choose a closed unbounded $C$ of $\lambda$ such that $\langle M \uparrow \gamma$, $\left.X_{b_{l}} \cap \gamma, X_{b_{l+1}} \cap \gamma, Z_{b_{l+1}} \cap \gamma\right\rangle$ is an elementary submodel of $\left\langle\mathcal{M}, X_{b_{l}}\right.$, $\left.X_{b_{l+1}}, Z_{b_{l+1}}\right\rangle$ for every $\gamma \in C$. Since $\left\langle b_{k} \uparrow \beta \mid k<l+2\right\rangle$ is in $T_{l+2}^{R}$ for every $\beta<\lambda, C_{b_{l+1}}$ must be a closed and unbounded subset of $\lambda$. Choose a limit ordinal $\delta$ from $C \cap C_{b_{l+1}}$. Let $t_{l}$ denote $b_{l}\left\lceil\delta\right.$ and $t_{l+1}$ denote $b_{l+1}\lceil\delta$. By the choice of the coding, $X_{t_{l}}=X_{b_{l}} \cap \delta, X_{t_{l+1}}=X_{b_{l+1}} \cap \delta$, and $Z_{t_{l+1}}=Z_{b_{l+1}} \cap \delta$. By the definition there should exist $X, Y, Z \subseteq \lambda$ such that $\langle\mathcal{M}, X, Y, Z\rangle \models \psi$ and $\left\langle M\left\lceil\delta, X_{t_{l}}, X_{t_{l+1}}, Z_{t_{l+1}}\right\rangle=\left\langle M \uparrow \delta, X_{b_{l}} \cap \delta, X_{b_{l+1}} \cap \delta, Z_{b_{l+1}} \cap \delta\right\rangle\right.$ is an elementary submodel of $\langle\mathcal{M}, X, Y, Z\rangle$.

So $\left\langle M\left\lceil\delta, X_{b_{l}} \cap \delta, X_{b_{l+1}} \cap \delta, Z_{b_{l+1}} \cap \delta\right\rangle\right.$ satisfies $\psi$, a contradiction.

4. A forcing notion increasing the well-ordering number. In this section $\lambda$ is an uncountable cardinal satisfying $\lambda^{<\lambda}=\lambda$. Our main target is Theorem 2 on page 209. For that we are going to define a forcing notion in two phases: first we define a basic version $\mathbb{P}^{-}(I)$ and then the real version $\mathbb{P}(I)$.

Definition 4.1. Suppose $I$ is a linear order. Let $\Gamma(I)$ denote the set of all finite (strictly) $I$-descending sequences of elements of $I$. For every $n<\omega$, the set $\{\eta \in \Gamma(I) \mid \operatorname{lh}(\eta)=n\}$ is abbreviated by $\Gamma_{n}(I)$.

Definition 4.2. Suppose $I$ is a linear order. The forcing notion $\mathbb{P}^{-}(I)$ consists of all the tuples $p=\left\langle\mathbf{T}^{p}, \Gamma^{p},\left\langle\mathbf{b}_{\eta}^{p} \mid \eta \in \Gamma^{p}\right\rangle\right\rangle$ satisfying the following demands: 
- $\mathbf{T}^{p}$ is an $\omega$-sequence $\left\langle T_{n}^{p} \mid n<\omega\right\rangle$ of $\lambda$-trees such that $\operatorname{ht}\left(\mathbf{T}^{p}\right)=\delta^{p}+1$ for some $\delta^{p} \in \lambda$;

- $\operatorname{card}\left(T_{n}^{p}\right)<\lambda$ for every $n<\omega$;

- $\Gamma^{p}$ is a subset of $\Gamma(I)$ such that $\operatorname{card}\left(\Gamma^{p}\right)<\lambda$ and $\Gamma^{p}$ is closed under initial segments $\left(\Gamma_{n}^{p}\right.$ denotes $\Gamma^{p} \cap \Gamma_{n}(I)$ for every $\left.n<\omega\right)$;

- for every $n<\omega$ and $\eta \in \Gamma_{n}^{p}, \mathbf{b}_{\eta}^{p} \in \operatorname{Br}\left(T_{n}^{p}\right)$ (remember that by Definition 3.2, $\left.\operatorname{Br}\left(T_{n}^{p}\right)=\left\{\mathbf{t} \in T_{n}^{p} \mid \operatorname{ht}(\mathbf{t})=\delta^{p}\right\}\right)$;

- for all $\eta \subseteq \nu \in \Gamma^{p}, \mathbf{b}_{\eta}^{p} \subseteq \mathbf{b}_{\nu}^{p}$.

The order of $\mathbb{P}^{-}(I)$ is defined by setting $q \leq p$ if

- $\operatorname{ht}\left(\mathbf{T}^{p}\right) \leq \operatorname{ht}\left(\mathbf{T}^{q}\right)$;

- for every $n<\omega, T_{n}^{q}$ is an end-extension of $T_{n}^{p}$, i.e., $T_{n}^{p}=\left\{\mathbf{t} \in T_{n}^{q} \mid\right.$ $\left.\operatorname{ht}(\mathbf{t})<\operatorname{ht}\left(T_{n}^{p}\right)\right\}$

- $\Gamma^{p} \subseteq \Gamma^{q}$

- $\mathbf{b}_{\eta}^{p} \unlhd \mathbf{b}_{\eta}^{q}$ for every $\eta \in \Gamma^{p}$.

Definition 4.3. Suppose $I$ is a linear order and $G$ is a $\mathbb{P}^{-}(I)$-generic filter over $\mathrm{V}$. We denote by $\mathbf{T}^{G}$ the sequence $\left\langle T_{n}^{G} \mid n<\omega\right\rangle$ where each $T_{n}^{G}$ is $\bigcup_{p \in G} T_{n}^{p}$. Moreover, $\mathbf{b}_{\emptyset}^{G}$ denotes $\emptyset$, and for every non-zero $n<\omega$ and $\eta \in \Gamma_{n}(I), \mathbf{b}_{\eta}^{G}$ denotes the sequence $\left\langle c_{l} \mid l<n\right\rangle$ where each $c_{l}$ is a union of all the $l$ th members of $\mathbf{b}_{\eta}^{p}$ for different $p$ 's in $G$.

To be sure that the definitions above make sense we note:

FACT 4.4. Suppose $I$ is a linear order and $\lambda^{<\lambda}=\lambda$.

(a) $\mathbb{P}^{-}(I)$ has $\lambda^{+}$-c.c.

(b) $\mathbb{P}^{-}(I)$ is $\lambda$-complete.

(c) For the rest of the items suppose $G$ is a $\mathbb{P}^{-}(I)$-generic filter over $\mathrm{V}$. In $\mathrm{V}[G], \mathbf{T}^{G}$ is an $\omega$-sequence of $\lambda$-trees having height $\lambda$.

(d) In $\mathrm{V}[G], \mathbf{b}_{\eta}^{G}$ is well defined for every $\eta \in \Gamma(I)$ and $\mathbf{b}_{\eta}^{G} \neq \mathbf{b}_{\nu}^{G} \in$ $\operatorname{Br}\left(T_{n}^{G}\right)$ for all $n<\omega$ and $\eta \neq \nu \in \Gamma_{n}(I)$. Moreover, for all $\eta, \nu \in$ $\Gamma(I)$,

$$
\mathbf{b}_{\eta}^{G} \subseteq \mathbf{b}_{\nu}^{G} \quad \text { if and only if } \quad \eta \subseteq \nu .
$$

(e) Suppose I is a well-ordering of order type at least $\alpha$. Then $\operatorname{rk}\left(\mathbf{T}^{G}\right) \geq$ $\alpha+1$ in $\mathrm{V}[G]$.

(f) In particular, if $I$ is a well-ordering of order type $\alpha, \operatorname{rk}\left(\mathbf{T}^{G}\right)$ is exactly $\alpha+1$ in $\mathrm{V}[G]$.

Proof. (a) This is a standard $\Delta$-lemma argument.

(b) This follows from the definition.

(c) By (b) the set $\left\{q \in \mathbb{P}^{-}(I) \mid \operatorname{ht}\left(T_{n}^{q}\right) \geq \delta\right\}$ is dense for every non-zero $n<\omega$ and $\delta<\lambda$. Hence the claim follows from the definition of $G$ and Definition 4.2. 
(d) The set $\left\{p \in \mathbb{P}^{-}(I) \mid \eta \in \Gamma^{p}\right\}$ is dense for every $\eta \in \Gamma(I)$. Hence the fact that $\mathbf{b}_{\eta}^{G}$ and $\mathbf{b}_{\nu}^{G}$ are branches through $T_{n}^{G}$ follows from Definition 4.2. Necessarily $\mathbf{b}_{\eta}^{G} \neq \mathbf{b}_{\nu}^{G}$ since $\left\{p \in \mathbb{P}^{-}(I) \mid \mathbf{b}_{\eta}^{p} \neq \mathbf{b}_{\nu}^{p}\right\}$ is dense when $\eta \neq \nu$. The last property follows from Definition 4.2 and the fact that whenever $\eta \nsubseteq \nu$, $\left\{p \in \mathbb{P}^{-}(I) \mid \mathbf{b}_{\eta}^{p} \nsubseteq \mathbf{b}_{\nu}^{p}\right\}$ is dense.

(e) Applying Definition 3.3 and (d) one can prove by induction on $\beta<\alpha$ that if $x_{\beta}$ is an element in $I$ such that $\left\{y \in I \mid y<_{I} x_{\beta}\right\}$ has order type $\beta$, then $\operatorname{rk}_{\mathbf{T}^{G}}\left(\mathbf{b}_{\left\langle x_{\beta}\right\rangle}^{G}\right) \geq \beta$. Hence $\operatorname{rk}_{\mathbf{T}^{G}}\left(\mathbf{b}_{\emptyset}^{G}\right) \geq \alpha+1$ (of course $\mathbf{b}_{\emptyset}^{G}$ is $\emptyset$ ).

(f) A proof of this fact is presented later on in Lemma 4.9 (for another reason we have to add some restrictions to $\left.\mathbb{P}^{-}(I)\right)$.

So far the forcing under consideration is a kind of $\omega$ copies of the standard Kurepa forcings (a Kurepa forcing adds a generic $\lambda$-tree with many $\lambda$-branches through it; cf. [Jec71] or [SV02, Lemma 2.3]). Of course the $\Gamma(I)$-structure makes the forcing a little bit more complicated. Our main goal is to have a generic extension where $2^{\lambda}$ is huge and the possible ranks of $\omega$-sequences of $\lambda$-trees are under very strict control (and thereby we know the value of $\delta(\lambda)$, see Theorem 2). Even though we do know the rank of the generic $\omega$-sequence $\mathbf{T}^{G}$ of $\lambda$-trees, we do not know whether $\mathbb{P}^{-}(\alpha)$ (or a product of such forcings) adds some new $\omega$-sequence of $\lambda$-trees having some unwanted rank. Hence we will introduce a modified version $\mathbb{P}(I)$ of the forcing $\mathbb{P}^{-}(I)$. The new properties of the modification are exploited in the next section. The main benefit is Lemma 5.1 which guarantees certain absoluteness between $\mathbb{P}(J)$ and $\mathbb{P}(I)$ for $J \subseteq I$. We postpone Lemma 5.1 to the next section in order to emphasize the fact that the modification is not yet applied in this section. On the contrary, we first want to demonstrate that the modification does not destroy the good properties of $\mathbb{P}^{-}(I)$.

The reader may look at the comment at the end of the proof of Lemma 5.1 if she or he has difficulties to see why the modification below is needed.

REMARK. In the next definition we would like to have "a potential

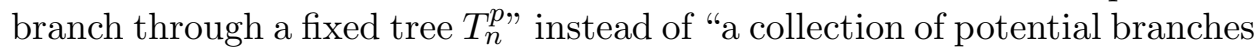
through all the trees $T_{n}^{p}, n<\omega$ ". However, our goal is to prove Lemma 5.1. Without considering " $\omega$-sequences of potential branches" Lemma 5.1 holds only for well-orderings, and that does not suffice for our purposes.

Definition 4.5. Suppose $I$ is a linear order. The conditions in the forcing $\mathbb{P}(I)$ are of the form

$$
p=\left\langle\mathbf{T}^{p}, \Gamma^{p},\left\langle\mathbf{b}_{\eta}^{p} \mid \eta \in \Gamma^{p}\right\rangle, \Delta^{p}\right\rangle,
$$

where $\mathbf{T}^{p}, \Gamma^{p}$, and $\left\langle\mathbf{b}_{\eta}^{p} \mid \eta \in \Gamma^{p}\right\rangle$ are as in Definition 4.2 and $\Delta^{p}$ is a set of pairs $\langle\eta, \mathbf{t}\rangle$ (t is "a potential branch", possibly of length $\omega$ ) such that

- $\operatorname{card}\left(\Delta^{p}\right)<\lambda$; 
- $\eta \in \Gamma^{p}$

- $\mathbf{t}=\left\langle t_{n} \mid n<n^{*}\right\rangle$, where $n^{*}$ is such that $\operatorname{lh}(\eta)<n^{*} \leq \omega$;

- $\mathbf{t} \mid n \in T_{n}^{p}$ for every $n<n^{*}$ (remember $\operatorname{Br}\left(T_{n}^{p}\right) \subseteq T_{n}^{p}$ because $\operatorname{ht}\left(T_{n}^{p}\right)$ is a successor ordinal).

Moreover, our main demand is that there exists a sequence $\mathbf{c}=\left\langle c_{n}\right|$ $\left.n<n^{*}\right\rangle$ such that

- $\mathbf{c}\left\lceil n \in \operatorname{Br}\left(T_{n}^{p}\right)\right.$ and $\mathbf{t} \unlhd \mathbf{c}\left\lceil n\right.$ for every finite $n \leq n^{*}$;

- $\mathbf{c}\left\lceil\operatorname{lh}(\eta)=\mathbf{b}_{\eta}^{p}\right.$.

To define the order $q \leq p$ for the elements of $\mathbb{P}(I)$ we demand, in addition to the demands presented in Definition 4.2, that $\Delta^{p} \subseteq \Delta^{q}$.

Remark. Even though $\mathbb{P}^{-}(I)$ is $\lambda$-complete, $\mathbb{P}(I)$ is not $\lambda$-complete anymore. However, $\mathbb{P}(I)$ is almost $\lambda$-complete as proved in Lemma 4.7.

We denote by Even the class of all even ordinals, i.e., Even $=\{\alpha \in$ On $\mid \alpha=0$ or $\alpha=\beta+2 n$ for some limit ordinal $\beta$ and $n<\omega\}$ (+ denotes the ordinal addition). The class of all odd ordinals, denoted by Odd, is On $\backslash$ Even.

Definition 4.6. Suppose $\langle\mathbb{P}, \leq\rangle$ is a partial order and $\mathbf{1}$ is its greatest element. Let $\theta$ be an ordinal. We denote by $\mathcal{G}_{\theta}(\mathbb{P})$ the following two-player Banach-Mazur game. The players, player 1 (also called "outward" player) and player 2 (also called "inward" player), choose in turns a sequence $\left\langle p_{i}\right|$ $i<\theta\rangle$ of elements in $\mathbb{P}$ such that

- $p_{0}$ is $\mathbf{1}$;

- if $i \in \theta \cap$ Odd, player 1 chooses an element $p_{i+1}$ such that $p_{i+1} \leq p_{i}$;

- if $i \in \theta \cap$ Even is nonzero, player 2 chooses an element $p_{i}$ such that $p_{i} \leq p_{j}$ for all $j<i$.

Player 2 wins a play of $\mathcal{G}_{\theta}(\mathbb{P})$ if he is able to follow the rules $\theta$ rounds. A partial order $\mathbb{P}$ is called strategically $\theta$-complete if player 2 has a winning strategy in the game $\mathcal{G}_{\theta}(\mathbb{P})$.

Lemma 4.7. For all linear orders $I, \mathbb{P}(I)$ is strategically $\lambda$-complete.

Proof. We describe a winning strategy for player 2. Suppose $i \in \lambda \cap$ Even is nonzero and the conditions $\left\langle p_{j} \mid j<i\right\rangle$ are already played. If $i$ is a successor ordinal, say $j+1$, player 2 chooses $p_{i}$ to be the condition $\left\langle\mathbf{T}^{p_{j}}, \Gamma^{p_{j}},\left\langle\mathbf{b}_{\eta}^{p_{j}} \mid \eta \in \Gamma^{p_{j}}\right\rangle, \Delta^{p_{i}}\right\rangle$, where

$$
\Delta^{p_{i}}=\Delta^{p_{j}} \cup\left\{\left\langle\eta, \mathbf{e}_{\eta, \mathbf{t}}^{i}\right\rangle \mid\langle\eta, \mathbf{t}\rangle \in \Delta^{p_{j}}\right\}
$$

and the extensions $\mathbf{e}_{\eta, \mathbf{t}}^{i}$ of $\mathbf{t}$ 's are defined as follows (remember that $\mathbf{t}$ might have length $\omega)$. For each $\langle\eta, \mathbf{t}\rangle \in \Delta^{p_{i}}\left(=\Delta^{p_{j}}\right)$, $\mathbf{e}_{\eta, \mathbf{t}}^{i}=\left\langle e_{n} \mid n<\operatorname{lh}(\mathbf{t})\right\rangle$ is such that 
(A) $\left\langle e_{m} \mid m<n\right\rangle \in \operatorname{Br}\left(T_{n}^{p_{i}}\right)$ for every finite $n \leq \operatorname{lh}(\mathbf{t})$;

(B) $t_{n}=e_{n}\left\lceil\operatorname{dom}\left(t_{n}\right)\right.$ for every $n<\operatorname{lh}(\mathbf{t})$;

(C) $\left\langle e_{n} \mid n<\operatorname{lh}(\eta)\right\rangle=\mathbf{b}_{\eta}^{p_{i}}$.

The existence of such objects is guaranteed by the definition of $\Delta^{p_{i}}$. The idea is that player 2 adds new potential branches extending the old ones. This ensures that in the forthcoming limit rounds player 2 is able to find witnessing branches for all "potential branches" introduced so far.

Suppose $i$ is a limit ordinal. Let $T_{n}^{*}$ denote $\bigcup_{j<i} T_{n}^{p_{j}}$ for every $n<\omega$. Let $\Delta^{*}$ denote $\bigcup_{j<i} \Delta^{p_{j}}$. Define $\Gamma^{*}$ to be $\bigcup_{j<i} \Gamma^{p_{j}}$, and $\mathbf{b}_{\eta}^{*}$ to be $\bigcup\left\{\mathbf{b}_{\eta}^{p_{j}} \mid\right.$ $j<i$ and $\left.\eta \in \Gamma^{p_{j}}\right\}$ for every $\eta \in \Gamma^{*}$. Fix $\langle\eta, \mathbf{t}\rangle$ from $\Delta^{*}$ for a while. By the choice of the strategy for player 2 in the earlier rounds, there exists a sequence $\mathbf{e}_{\eta, \mathbf{t}}^{i}=\left\langle e_{n} \mid n<\operatorname{lh}(\mathbf{t})\right\rangle$ such that $\left\langle e_{m} \mid m<n\right\rangle \in \operatorname{Br}\left(T_{n}^{*}\right)$ for every finite $n<\operatorname{lh}(\mathbf{t})$, and also (B) and (C) are satisfied when $\mathbf{b}_{\eta}^{p_{j}}$ is replaced with $\mathbf{b}_{\eta}^{*}$. Now player 2 chooses $p_{i}$ to be $\left\langle\mathbf{T}^{p_{i}}, \Gamma^{p_{i}},\left\langle\mathbf{b}_{\eta}^{p_{i}} \mid \eta \in \Gamma^{p_{i}}\right\rangle, \Delta^{p_{i}}\right\rangle$, where

- $\Gamma^{p_{i}}=\Gamma^{*}$

- $\mathbf{b}_{\eta}^{p_{i}}=\mathbf{b}_{\eta}^{*}$ for every $\eta \in \Gamma^{p_{i}}$

- $\Delta^{p_{i}}=\left\{\left\langle\eta, \mathbf{e}_{\eta, \mathbf{t}}^{i}\right\rangle \mid\langle\eta, \mathbf{t}\rangle \in \Delta^{*}\right\} \cup \Delta^{*}$;

- $T_{n}^{p_{i}}=T_{n}^{*} \cup\left\{\mathbf{b}_{\eta}^{p_{i}} \mid \eta \in \Gamma^{p_{i}}\right\} \cup\left\{\mathbf{e}_{\eta, \mathbf{t}}^{i} \mid\langle\eta, \mathbf{t}\rangle \in \Delta^{*}\right\}$ for every $n<\omega$;

- $\mathbf{T}^{p_{i}}=\left\langle T_{n}^{p_{i}} \mid n<\omega\right\rangle$.

Lemma 4.8. Suppose $I$ is a linear order and $G$ is a $\mathbb{P}(I)$-generic filter over V. Let $\mathbf{T}^{G}$ and $\mathbf{b}_{\eta}^{G}$ for $\eta \in \Gamma(I)$ be as in Definition 4.3 .

(a) $\mathbf{T}^{G}$ is an $\omega$-sequence of $\lambda$-trees in $\mathrm{V}[G]$ and $\operatorname{ht}\left(\mathbf{T}^{G}\right)=\lambda$.

(b) For all $n<\omega$ and $\eta \neq \nu \in \Gamma_{n}(I), \mathbf{b}_{\eta}^{G} \neq \mathbf{b}_{\nu}^{G} \in \operatorname{Br}\left(T_{n}^{G}\right)$. Moreover, for all $\eta, \nu \in \Gamma(I), \mathbf{b}_{\eta}^{G} \subseteq \mathbf{b}_{\nu}^{G}$ if and only if $\eta \subseteq \nu$.

(c) If $I$ is a well-ordering of order type $\alpha$, then $\operatorname{rk}\left(\mathbf{T}^{G}\right) \geq \alpha+1$ in $\mathrm{V}[G]$.

Proof. (a) Suppose $\delta$ is a limit ordinal below $\lambda$ and $p \in \mathbb{P}(I)$. By Lemma 4.7, player 2 can use his winning strategy against such a move of player 1 that after $\delta$ rounds $p_{1}=p$ and $T_{n}^{p_{i+1}} \neq T_{n}^{p_{i}}$ for all $0<n<\omega$ and $0<i<\delta$. Hence ht $\left(\mathbf{T}^{p_{\delta}}\right) \geq \delta$ where $p_{\delta}$ is the answer of player 2 in round $\delta$. Applying this idea we conclude that $\left\{q \in \mathbb{P}(I) \mid \operatorname{ht}\left(T_{n}^{q}\right) \geq \delta\right\}$ is dense for every nonzero $n<\omega$ and $\delta<\lambda$.

The rest of the items are proved in the same manner as Fact 4.4.

Lemma 4.9. Suppose $I$ is a well-ordering of order type $\alpha$ and $G$ is a $\mathbb{P}(I)$-generic filter over $\mathrm{V}$. Moreover, suppose $\widetilde{\mathbb{Q}}$ is a $\mathbb{P}(I)$-name for a forcing notion such that

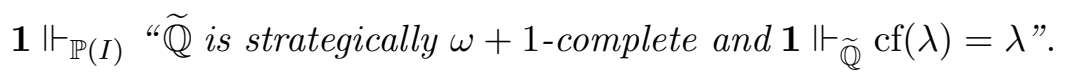

Let $H$ be a $\widetilde{\mathbb{Q}}^{G}$-generic filter over $\mathrm{V}[G]$. Then $\mathbf{T}^{G}$ has rank $\alpha+1$ in $\mathrm{V}[G][H]$. 
Proof. By Lemma 4.8(c), $\operatorname{rk}\left(\mathbf{T}^{G}\right) \geq \alpha+1$ in $\mathrm{V}[G]$ and, by the absoluteness of the $\Sigma_{1}^{1}$-relations, the same holds in $\mathrm{V}[G][H]$. Without loss of generality $I$ equals $\langle\alpha, \in\rangle$. It remains to prove $\operatorname{rk}_{\mathbf{T}^{G}}(\emptyset) \nsupseteq \alpha+2$. By Lemma 4.8(b) it suffices to prove in $\mathrm{V}[G][H]$ that for all non-zero $n<\omega$,

$$
\operatorname{Br}\left(T_{n}^{G}\right)=\left\{\mathbf{b}_{\eta}^{G} \mid \eta \in \Gamma_{n}(I)\right\}
$$

Namely, applying (A) we reach a contradiction as follows. Suppose $\operatorname{rk}_{\mathbf{T}^{G}}\left(\mathbf{b}_{\emptyset}^{G}\right) \geq \alpha+2$. Define $\alpha_{0}=\alpha+1$ and choose $\alpha_{1}<\alpha_{0}$ such that $\operatorname{rk}_{\mathbf{T}^{G}}\left(\mathbf{b}_{\left\langle\alpha_{1}\right\rangle}^{G}\right) \geq \alpha_{0}$. If $\alpha_{1}>0$, we may choose $\alpha_{2}<\alpha_{1}$ such that $\operatorname{rk}_{\mathbf{T}^{G}}\left(\mathbf{b}_{\left\langle\alpha_{1}, \alpha_{2}\right\rangle}^{G}\right)$ $\geq \alpha_{1}$. We may continue choosing $\alpha_{m}$ 's until for some $n<\omega, \alpha_{n+1}=0$ and $\operatorname{rk}_{\mathbf{T}^{G}}\left(\mathbf{b}_{\eta}^{G}\right) \geq \alpha_{n}>0$, where $\eta=\left\langle\alpha_{1}, \ldots, \alpha_{n+1}\right\rangle$. Hence there should be $\mathbf{c} \in \operatorname{Br}\left(T_{n+2}^{G}\right)$ such that $\mathbf{c}\left\lceil(n+1)=\mathbf{b}_{\eta}^{G}\right.$. By (A) there should be $\nu \in \Gamma_{n+2}(I)$ satisfying $\mathbf{c}=\mathbf{b}_{\nu}^{G}$, and moreover, by Lemma $4.8(\mathrm{~b}), \eta \subsetneq \nu$. This contradicts the fact that $\nu$ should be a strictly descending sequence of ordinals.

We prove (A) in the same manner as an analogous lemma is proved for Kurepa trees in [Jec71] (or in [SV02, Lemma 2.3]). The only difference is that instead of " $\lambda$-completeness" we apply "strategical $\omega+1$-completeness of $\widetilde{\mathbb{Q}}$ " together with "a special winning strategy" for player 2 in $\mathcal{G}_{\omega+1}(\mathbb{P}(I))$. The winning strategy described in the proof of Lemma 4.7 does not suffice, because in addition to "avoiding the generic branches" we are forced to "avoid the potential branches extended by the winning strategy" (see (F) below).

Suppose $n^{*}<\omega,\left\langle p_{0}, \widetilde{q_{0}}\right\rangle$ is a condition in $\mathbb{P}(I) * \widetilde{\mathbb{Q}}$, and $\widetilde{\mathbf{c}}$ is a name such that

$$
\left\langle p_{0}, \widetilde{q_{0}}\right\rangle \Vdash \widetilde{\mathbf{c}} \in \operatorname{Br}\left(T_{n^{*}}^{\widetilde{G}}\right) \backslash\left\{\mathbf{b}_{\eta}^{\widetilde{G}} \mid \eta \in \Gamma_{n^{*}}(I)\right\} .
$$

Moreover, let $\widetilde{w}$ be a $\mathbb{P}(I)$-name such that

$\left\langle p_{0}, \widetilde{q}_{0}\right\rangle \Vdash$ " $\widetilde{w}$ is a winning strategy for player 2 in $\mathcal{G}_{\omega+1}(\widetilde{\mathbb{Q}}) "$.

Let $\delta_{0}$ be the height of $T_{n^{*}}^{p_{0}}$. By induction on $0<i<\omega$ we define conditions $\left\langle p_{i}, \widetilde{q}_{i}\right\rangle \in \mathbb{P}(I) * \widetilde{\mathbb{Q}}$ as follows. Always $\delta_{i}$ is a shorthand for $\operatorname{ht}\left(T_{n^{*}}^{p_{i}}\right)$.

For $i \in \omega \cap \operatorname{Odd}$, say $i=j+1,\left\langle p_{i}, \widetilde{q}_{i}\right\rangle$ is such that

$$
\begin{gathered}
p_{i} \leq p_{j}, \\
p_{i} \Vdash \widetilde{q}_{i} \leq \widetilde{q}_{j}, \\
\operatorname{ht}\left(T_{n^{*}}^{p_{i}}\right)>\operatorname{ht}\left(T_{n^{*}}^{p_{j}}\right), \\
\left\langle p_{i}, \widetilde{q}_{i}\right\rangle \Vdash \widetilde{\mathbf{c}} \downarrow \delta_{i} \notin\left\{\mathbf{b}_{\eta}^{p_{i}} \downarrow \delta_{i} \mid \eta \in \Gamma_{n^{*}}^{p_{j}}\right\} .
\end{gathered}
$$

There is no problem to find such a condition because we used $\Gamma_{n^{*}}^{p_{j}}$ instead of $\Gamma_{n^{*}}^{p_{i}}$ in (E) and by the assumption $\mathbf{1} \Vdash_{\mathbb{P}(I) * \widetilde{\mathbb{Q}}} \operatorname{cf}(\lambda)=\lambda$, every condition below $\left\langle p_{0}, \widetilde{q_{0}}\right\rangle$ forces that for every $X \subseteq \Gamma_{n^{*}}(I)$ of cardinality $<\lambda$ there is $\gamma$ below $\lambda$ such that

$$
\widetilde{\mathbf{c}} \downarrow \gamma \notin\left\{\mathbf{b}_{\eta}^{\widetilde{G}} \downarrow \gamma \mid \eta \in X\right\} .
$$


For non-zero $i \in \omega \cap$ Even, say $i=j+1,\left\langle p_{i}, \widetilde{q}_{i}\right\rangle$ is chosen as follows. First let $\langle r, \widetilde{s}\rangle \leq\left\langle p_{j}, \widetilde{q_{j}}\right\rangle$ be such that $\operatorname{ht}\left(T_{n^{*}}^{r}\right)=\delta_{j}+2$ and also the following holds. For every $\mathbf{t} \in \operatorname{Br}\left(T_{n^{*}}^{p_{j}}\right)$ and $\eta \in \Gamma_{m}^{p_{j}}, m<n^{*}$, if $\mathbf{b}_{\eta}^{p_{j}} \subseteq \mathbf{t}$ then there exist $\mathbf{c} \neq \mathbf{d}$ in $\operatorname{Br}\left(T_{n^{*}}^{r}\right)$ such that

- $\mathbf{t} \unlhd \mathbf{c}$ and $\mathbf{t} \unlhd \mathbf{d}$;

- $\langle\eta, \mathbf{c}\rangle$ and $\langle\eta, \mathbf{d}\rangle$ are in $\Delta^{r}$.

Then choose $\left\langle r^{\prime}, \widetilde{s}^{\prime}\right\rangle \leq\langle r, \widetilde{s}\rangle$ such that for some $\mathbf{s}_{i} \in \mathrm{V}$ (remember that $\mathbb{P}(I)$ does not add sets of cardinality $<\lambda$ ),

$$
\left\langle r^{\prime}, \widetilde{s}^{\prime}\right\rangle \Vdash \widetilde{\mathbf{c}} \downarrow\left(\delta_{j}+1\right)=\mathbf{s}_{i} \in T_{n^{*}}^{\widetilde{G}} .
$$

Choose $p_{i} \leq r$ as follows (as in the proof of Lemma 4.7 except that the height of $\mathbf{T}^{r}$ might increase and (F) below holds). Ensure that for each $\langle\eta, \mathbf{t}\rangle \in \Delta^{r}$ there exists $\mathbf{e}_{\eta, \mathbf{t}}^{i}$ such that (A), (B), and (C) from the proof of Lemma 4.7 are satisfied, and moreover,

$$
\text { if } \mathbf{t} \in T_{n^{*}}^{p_{j}} \text { and } \mathbf{t} \unlhd \mathbf{s}_{i} \text {, then } \mathbf{s}_{i} \unlhd \mathbf{e}_{\eta, \mathbf{t}}^{i} \text { (by the choice of ht }\left(\mathbf{s}_{i}\right), \mathbf{t} \neq \mathbf{s}_{i} \text { ). }
$$

This time there is no problem to fulfill these demands because of the choice of $r$ (and in (F) we used $T_{n^{*}}^{p_{j}}$ instead of $T_{n^{*}}^{r^{\prime}}$ ). As in the successor case of the proof of Lemma 4.7, make sure that $\Delta^{p_{i}}=\Delta^{r} \cup\left\{\left\langle\eta, \mathbf{e}_{\eta, \mathbf{t}}^{i}\right\rangle \mid\langle\eta, \mathbf{t}\rangle \in \Delta^{r}\right\}$. Choose $\widetilde{q}_{i}$ so that $p_{i} \Vdash_{\mathbb{P}(I)} \widetilde{w}\left(\left\langle\widetilde{q}_{j} \mid j<i\right\rangle\right)=\widetilde{q}_{i}\left(\widetilde{q}_{i}\right.$ is the answer of player 2 according to the winning strategy $\widetilde{w})$.

Now define $\left\langle p_{\omega}, \widetilde{q}_{\omega}\right\rangle$ as follows. The condition $p_{\omega}$ is defined as in the limit case of the proof of Lemma 4.7. Recall that $\Delta^{*}$ denotes $\bigcup_{i<\omega} \Delta^{p_{i}}$. Then we have

$$
\operatorname{Br}\left(T_{n^{*}}^{p_{\omega}}\right)=\left\{\mathbf{b}_{\eta}^{p_{\omega}} \mid \eta \in \Gamma^{p_{\omega}}\right\} \cup\left\{\mathbf{e}_{\eta, \mathbf{t}}^{i} \mid\langle\eta, \mathbf{t}\rangle \in \Delta^{*}\right\} .
$$

Define $\widetilde{q}_{\omega}$ to be such that $p_{\omega} \Vdash_{\mathbb{P}(I)} \widetilde{w}\left(\left\langle\widetilde{q}_{i} \mid i<\omega\right\rangle\right)=\widetilde{q}_{\omega}$. Then $\left\langle p_{\omega}, \widetilde{q}_{\omega}\right\rangle$ is a lower bound for the conditions $\left\langle p_{i}, \widetilde{q}_{i}\right\rangle, i<\omega$, and it forces $\widetilde{\mathbf{c}} \downarrow \delta_{\omega} \in \operatorname{Br}\left(T_{n^{*}}^{p_{\omega}}\right)$. On the other hand, by (E) we have $\widetilde{\mathbf{c}} \downarrow \delta_{\omega} \notin\left\{\mathbf{b}_{\eta}^{p_{\omega}} \mid \eta \in \Gamma^{p_{\omega}}\right\}$ and by (F) we have $\widetilde{\mathbf{c}} \downarrow \delta_{\omega} \notin\left\{\mathbf{e}_{\eta, \mathbf{t}}^{i} \mid \mathbf{t} \in T_{n^{*}}^{p_{\omega}}\right.$ and $\left.\langle\eta, \mathbf{t}\rangle \in \Delta^{*}\right\}$. This is a contradiction.

5. The well-ordering number can have large cofinality. The property introduced in the first lemma is the reason for the modification $\mathbb{P}(I)$ (Definition 4.5) of $\mathbb{P}^{-}(I)$. The property has a central role in the proof of Theorem 2 presented after some definitions.

Lemma 5.1. Suppose $J$ is a sublinear order of $I$. Then $\mathbb{P}(J)$ is a complete subforcing of $\mathbb{P}(I)$ (for a definition and properties of a complete subset see e.g. [Kun83, She98]).

Proof. We must show that every maximal antichain $A \subseteq \mathbb{P}(J)$ remains a maximal antichain in $\mathbb{P}(I)$. Clearly, if $p, q \in \mathbb{P}(J)$ and $r \in \mathbb{P}(I)$ are such that $r \leq p, q$, then the "reduction $r^{\prime}$ of $r$ to $J$ " satisfies $r^{\prime} \leq p, q$, where 
$r^{\prime}=\left\langle\mathbf{T}^{r}, \Gamma^{r^{\prime}},\left\langle\mathbf{b}_{\eta}^{r} \mid \eta \in \Gamma^{r^{\prime}}\right\rangle, \Delta^{r^{\prime}}\right\rangle, \Gamma^{r^{\prime}}=\Gamma^{r} \cap \Gamma(J)$, and $\Delta^{r^{\prime}}=\Delta^{r} \cap$
$\left(\Gamma(J) \times \bigcup_{n<\omega} T_{n}^{r}\right)$.

To prove that the maximality is preserved we show that for every $q \in$ $\mathbb{P}(I)$ there is a special reduction $q^{\prime \prime}$ to $J$ such that for every $p \leq q^{\prime \prime}$ in $\mathbb{P}(J)$ there exists $r \in \mathbb{P}(I)$ satisfying $r \leq p, q$. The special reduction $q^{\prime \prime}$ is like the reduction $q^{\prime}$ defined above except that the branches having index in $\Gamma^{q} \backslash \Gamma(J)$ are included into the set of potential branches of $q^{\prime \prime}$. The formal details are given below.

We define $q^{\prime \prime}=\left\langle\mathbf{T}^{q}, \Gamma^{q^{\prime \prime}},\left\langle\mathbf{b}_{\eta}^{q} \mid \eta \in \Gamma^{q^{\prime \prime}}\right\rangle, \Delta^{q^{\prime \prime}}\right\rangle$, where $\Gamma^{q^{\prime \prime}}=\Gamma^{q} \cap \Gamma(J)$ as in the "ordinary reduction". We define $\Delta^{q^{\prime \prime}}$ to contain $\Delta^{q} \cap\left(\Gamma(J) \times \bigcup_{n<\omega} T_{n}^{q}\right)$, but we also add some new members. Suppose $\eta$ and $\left\langle\nu_{m} \mid m<m^{*}\right\rangle$ are such that

- $1 \leq m^{*} \leq \omega$

- $\eta \in \Gamma(J) \cap \Gamma^{q}$ (remember $\left.\emptyset \in \Gamma(J)\right)$;

- $\nu_{m} \in \Gamma^{q} \backslash \Gamma(J)$ for every $m<m^{*}$;

- $\eta \subseteq \nu_{0}$ and $\operatorname{lh}\left(\nu_{0}\right)=\operatorname{lh}(\eta)+1$;

- $\nu_{m} \subseteq \nu_{m+1}$ and $\operatorname{lh}\left(\nu_{m+1}\right)=\operatorname{lh}\left(\nu_{m}\right)+1$ for every $m<m^{*}-1$;

- if $m^{*}$ is finite, $\nu$ is maximal in $\Gamma^{q}$ with respect to $\subseteq$.

For such objects we set $\langle\eta, \mathbf{t}\rangle \in \Delta^{q^{\prime \prime}}$, where $\mathbf{t}=\left\langle t_{n}\right| n\left\langle n^{*}\right\rangle$ is such that $n^{*}=\operatorname{lh}(\eta)+m^{*}$ if $m^{*}$ is finite, and $m^{*}=\omega$ otherwise, and moreover, for every finite $n \leq n^{*}$,

$$
\left\langle t_{l} \mid l<\operatorname{lh}\left(\nu_{n}\right)\right\rangle=\mathbf{b}_{\nu_{n}}^{q} .
$$

Now it is easy to check (since $\Delta^{q^{\prime \prime}} \subseteq \Delta^{p}$ ) that for given $p \leq q^{\prime \prime}$, every branch $\mathbf{b}_{\nu}^{q}$ with $\nu \in \Gamma^{q} \backslash \Gamma(J)$ can be extended to a branch through $T_{n}^{p}$. It follows that the desired condition $r=\left\langle\mathbf{T}^{r}, \Gamma^{r},\left\langle\mathbf{b}_{\eta}^{r} \mid \eta \in \Gamma^{r}\right\rangle, \Delta^{r}\right\rangle$ is such that $\mathbf{T}^{r}=\mathbf{T}^{p}, \Gamma^{r}=\Gamma^{p} \cup \Gamma^{q}, \Delta^{r}=\Delta^{p} \cup \Delta^{q}$, and the branches $\mathbf{b}_{\nu}^{r}, \nu \in \Gamma^{r}$, are chosen according to the demands. (The reader may wonder what goes wrong if $p \in \mathbb{P}^{-}(J)$. In that case consider two different branches $\mathbf{b}_{\nu}^{q}$ and $\mathbf{b}_{\varrho}^{q}$ with $\eta \subseteq \nu, \varrho \in \Gamma^{q} \backslash \Gamma(J)$. Then $p$ might be such that for say $\mathbf{b}_{\nu}^{q}$ there exists a branch $\mathbf{c}$ through $T_{n}^{p}$ with $\mathbf{c}\left\lceil\operatorname{lh}(\eta)=\mathbf{b}_{\eta}^{p}\right.$, because by the definition $\mathbf{b}_{\eta}^{p}$ must be an initial segment of some branch in $\operatorname{Br}\left(T_{n}^{p}\right)$. However $\mathbf{b}_{\varrho}^{q}$ might be such that no extension of it has $\mathbf{b}_{\eta}^{p}$ as its initial segment.)

Definition 5.2. We are going to use the following assumptions:

(A) $\quad \lambda$ is an uncountable cardinal such that $\lambda^{<\lambda}=\lambda$;

(B) $\theta$ is a cardinal such that $\operatorname{cf}(\theta) \geq \lambda^{+}$and $\xi^{\lambda}<\theta$ for every $\xi<\theta$;

(C) $\quad \kappa$ is a cardinal such that $\theta \leq \kappa$ and $\kappa^{\lambda}=\kappa$.

The following definition introduces an auxiliary device. We are going to force $\delta(\lambda)$ to have a desired value $\theta \in 2^{\lambda} \backslash \lambda^{+}$. So we first add generic $\omega$ sequences of $\lambda$-trees using $\mathbb{P}(\alpha)$ for every $\alpha<\theta$. Then we have to argue that there does not exist an $\omega$-sequence of $\lambda$-trees having rank between $\theta$ and $\infty$. 
To succeed in the latter task we need some auxiliary forcing. A solution is to force with $\mathbb{P}(I)$ 's where $I$ is not well-ordered, and to show that such a forcing adds a "descending" sequence of branches to any $\omega$-sequence of $\lambda$-trees of rank $\geq \theta$. Hence the forcings together ensure that " $\operatorname{rk}(\mathbf{T}) \geq \theta$ implies $\operatorname{rk}(\mathbf{T})=\infty$ " in the generic extension.

Definition 5.3. We define a linear order $\left\langle I_{\text {lex }},<_{\text {lex }}\right\rangle$ as follows. $I_{\text {lex }}$ consists of all finite sequences of ordinals below $\lambda^{+}$. For all $\bar{x}, \bar{y} \in I_{\text {lex }}$, define $\bar{x}<_{\operatorname{lex}} \bar{y}$ if

- $l=\operatorname{lh}(\bar{x})<\operatorname{lh}(\bar{y})$ and $\bar{x}=\bar{y} \uparrow l$, or

- there exists $l<\min \{\operatorname{lh}(\bar{x}), \operatorname{lh}(\bar{y})\}$ such that $\bar{x}|l=\bar{y}| l$ and $x_{l}<y_{l}$.

Definition 5.4. Let $\left\langle\alpha_{i}^{*} \mid i<\theta\right\rangle$ be an increasing enumeration of all limit ordinals below $\theta$. Define $\overline{\mathbb{P}}(\bar{I})$ to be the product of the forcings in $\left\langle\mathbb{P}\left(I_{i}\right) \mid i<\kappa\right\rangle$ with $<\lambda$-support, where

- for every $i<\theta, I_{i}$ is $\left\langle\alpha_{i}^{*}, \in\right\rangle$;

- for every $i \in \kappa \backslash \theta, I_{i}$ is $\left\langle I_{\text {lex }},<_{\text {lex }}\right\rangle$.

TheORem 2. Suppose $\lambda<\theta \leq \kappa$ are cardinals as in Definition 5.2 and $\overline{\mathbb{P}}(\bar{I})$ is a forcing as in Definition 5.4. In $\mathrm{V}^{\overline{\mathbb{P}}(\bar{I})}$ we have:

- all cofinalities are preserved;

- no new sets of cardinality $<\lambda$ are added;

- $\lambda^{<\lambda}=\lambda$;

- $2^{\lambda}=\kappa$

- $\delta(\lambda)=\theta$.

The rest of this section is dedicated to the proof of this theorem.

The first two items follow from Lemma 4.7, Lemma 4.8, and the fact that $\overline{\mathbb{P}}(\bar{I})$ has $\lambda^{+}$-c.c. Moreover, $2^{\lambda} \geq \kappa$ in $\mathrm{V}^{\overline{\mathbb{P}}(\bar{I})}$, because each $\mathbb{P}\left(I_{i}\right)$ adds a new subset of $\lambda$, and $2^{\lambda} \leq \kappa$ in $\mathrm{V}^{\overline{\mathbb{P}}(\bar{I})}$, because $\operatorname{card}(\overline{\mathbb{P}}(\bar{I}))=\kappa, \overline{\mathbb{P}}(\bar{I})$ has $\lambda^{+}$-c.c., and we have assumed Definition 5.2(C).

By Lemma 3.4, $\delta(\lambda)$ equals $\theta$ if in $\mathrm{V}^{\overline{\mathbb{P}}(\bar{I})}$ we have

(5.1) for every $\alpha<\theta$ there is an $\omega$-sequence $\mathbf{T}$ of $\lambda$-trees such that $\alpha \leq$ $\operatorname{rk}(\mathbf{T}) \neq \infty$;

(5.2) for every $\omega$-sequence $\mathbf{T}$ of $\lambda$-trees, either $\operatorname{rk}(\mathbf{T})<\theta$ or $\operatorname{rk}(\mathbf{T})=\infty$.

Since $\overline{\mathbb{P}}(i)$ does not add new sets of cardinality $<\lambda$,

$$
\mathbf{1} \Vdash_{\overline{\mathbb{P}}(i)} \text { " } \mathbb{P}\left(I_{i}\right) \text { is strategically } \lambda \text {-complete" }
$$

for every $i<\kappa$. Thus Lemma 4.9 guarantees that in $\mathrm{V}^{\overline{\mathbb{P}}(\bar{I})}$, for every $i \in \theta$ there is an $\omega$-sequence $\mathbf{T}^{i}$ of $\lambda$-trees such that $\operatorname{rk}\left(\mathbf{T}^{i}\right)=\operatorname{rk}\left(I_{i}\right)=\alpha_{i}^{*}$. It follows that (5.1) holds in $\mathrm{V}^{\overline{\mathbb{P}}(\bar{I})}$. 
It remains to prove $(5.2)$ in $\mathrm{V}^{\overline{\mathbb{P}}(\bar{I})}$. Suppose $p^{*} \in \overline{\mathbb{P}}(\bar{I})$ and $\left\langle\widetilde{T}_{n} \mid n<\omega\right\rangle$ are $\overline{\mathbb{P}}(\bar{I})$-names such that

$$
p^{*} \Vdash \text { " }\left\langle\widetilde{T}_{n} \mid n<\omega\right\rangle \text { is an } \omega \text {-sequence of } \lambda \text {-trees having rank } \geq \theta . "
$$

To simplify our notation let $\widetilde{\mathbf{T}}$ be a $\overline{\mathbb{P}}(\bar{I})$-name such that

$$
\mathbf{1} \Vdash \widetilde{\mathbf{T}}=\left\langle\widetilde{T}_{n} \mid n<\omega\right\rangle .
$$

We aim at the conclusion $p^{*} \Vdash \operatorname{rk}(\widetilde{\mathbf{T}})=\infty$.

Since $\overline{\mathbb{P}}(I)$ does not add new functions having domain of cardinality $<\lambda$, the interpretation of each $\widetilde{T}_{n}$ in a generic extension is a subset of V. Moreover, because of the $\lambda^{+}$-c.c. property, we may assume that each $\operatorname{dom}\left(\widetilde{T}_{n}\right)$ is a nice $\overline{\mathbb{P}}(\bar{I})$-name of cardinality at most $\lambda$.

Even though our proof is quite straightforward, we want to introduce some notations in order to be precise.

Definition 5.5. For a $\overline{\mathbb{P}}(\bar{I})$-name $\sigma$, let $\operatorname{dom}(\sigma)$ denote the smallest subset $X$ of $\kappa$ such that $\operatorname{dom}(p) \subseteq X$ for all conditions $p$ appearing in $\sigma$. For every $p \in \overline{\mathbb{P}}(I)$, define $\bar{I}^{p}$ to be the sequence $\left\langle I_{i}^{p} \mid i \in \operatorname{dom}(p)\right\rangle$, where $I_{i}^{p}$ is $\left\{x \in I_{i} \mid\right.$ for some $\eta \in \Gamma^{p(i)}, x$ appears in $\left.\eta\right\}$. Moreover, let $\bar{I}^{\sigma}$ denote the sequence $\left\langle I_{i}^{\sigma} \mid i \in \operatorname{dom}(\sigma)\right\rangle$, where $I_{i}^{\sigma}=\left\{x \in I_{i} \mid\right.$ for some $p$ appearing in $\sigma, i \in \operatorname{dom}(p)$ and $\left.x \in I_{i}^{p}\right\}$.

Definition 5.6. A sequence $\bar{J}=\left\langle J_{i} \mid i \in \operatorname{dom}(\bar{J})\right\rangle$ is called a part of $\bar{I}$ if $\operatorname{dom}(\bar{J}) \subseteq \operatorname{dom}(\bar{I})=\kappa$ and $J_{i}$ is a non-empty subset of $I_{i}$ for every $i \in \operatorname{dom}(\bar{J})$. Such a sequence is called a small part of $\bar{I}$ if $\operatorname{card}(\operatorname{dom}(\bar{J})) \leq \lambda$, and $\operatorname{card}\left(J_{i}\right) \leq \lambda$ for every $i \in \operatorname{dom}(\bar{J}) \cap \theta$ (for $i \in \kappa \backslash \theta$, card $\left(J_{i}\right)$ might be $\left.\lambda^{+}\right)$.

REMARK. For every nice name $\sigma$ for a subset of $\mathrm{V}$ of cardinality at most $\lambda, \bar{I}^{\sigma}$ is a small part of $\bar{I}$. Without loss of generality also $\widetilde{\mathbf{T}}$ is chosen so that $\bar{I}^{\widetilde{\mathbf{T}}}$ and each $\bar{I}^{\widetilde{T}_{n}}$ are small parts of $\bar{I}$.

For parts $\bar{J}$ of $\bar{I}$ let $\overline{\mathbb{P}}(\bar{J})$ denote the product of the forcings $\mathbb{P}\left(J_{i}\right), i \in$ $\operatorname{dom}(\bar{J})$, with $<\lambda$-support.

FACT 5.7. For every part $\bar{J}$ of $\bar{I}, \overline{\mathbb{P}}(\bar{J})$ is a complete subforcing of $\overline{\mathbb{P}}(\bar{I})$.

Proof. This is a standard consequence of Lemma 5.1.

Before the introduction of the main tool we need two more auxiliary definitions.

Definition 5.8. Suppose $\mathbf{J}$ is a finite sequence of small parts of $\bar{I}$. Let $\operatorname{dom}(\mathbf{J}) \operatorname{denote} \bigcup_{\bar{J} \in \mathbf{J}} \operatorname{dom}(\bar{J})$ and $\mathbf{J}[i] \operatorname{denote} \bigcup\left\{J_{i} \mid \bar{J} \in \mathbf{J}\right.$ and $\left.i \in \operatorname{dom}(\bar{J})\right\}$ for every $i \in \operatorname{dom}(\mathbf{J})$. By $\overline{\mathbb{P}}(\mathbf{J})$ we denote the forcing $\overline{\mathbb{P}}(\langle\mathbf{J}[i] \mid i \in \operatorname{dom}(\mathbf{J})\rangle)$. 
Definition 5.9. Define a part $\bar{K}=\left\langle K_{i} \mid i<\operatorname{dom}(\bar{K})\right\rangle$ of $\bar{I}$ by setting

- $\operatorname{dom}(\bar{K})=\operatorname{dom}\left(p^{*}\right) \cup \operatorname{dom}(\widetilde{\mathbf{T}}) \cup\left(\left(\theta+\lambda^{+}\right) \backslash \theta\right)$ (where the + after the first $\theta$ denotes the ordinal addition);

- $K_{i}=I_{i}=\alpha_{i}^{*}$ if $i \in \operatorname{dom}(\bar{K}) \cap \theta$;

- $K_{i}=I_{\text {lex }}$ if $i \in \operatorname{dom}(\bar{K}) \backslash \theta$.

The next definition might look a little bit technical but the content of it is very simple: we introduce an order-preserving mapping which determines a complete embedding from $\overline{\mathbb{P}}(\mathbf{J})$ into $\overline{\mathbb{P}}(\bar{K})$ (for a definition and properties of a complete embedding see e.g. [Kun83, She98]). The source of the technicality is the need that the mapping must be "extendable" when the length of the finite sequence of small parts increases.

Definition 5.10. For all finite sequences $\mathbf{J}$ of small parts of $\bar{I}$, we define "order preserving" mappings o "from $\mathbf{J}$ into $\bar{K}$ " by induction on $\operatorname{lh}(\mathbf{J})<\omega$ as follows. Set $\mathrm{o}^{\emptyset}=\emptyset$. Suppose $\mathrm{o}$ is already defined and $\bar{J}$ is a small part of $\bar{I}$. A mapping o $\mathbf{J} \frown\langle\bar{J}\rangle$ is defined as follows.

Without loss of generality we may assume that each set $J$ appearing in $\mathbf{J}$ or $\bar{J}$ is closed under supremums. (If that is not the case, take a closure. Then the resulting parts remain small.)

Let $\operatorname{dom}\left(\mathrm{o}^{\mathbf{J}}\langle\langle\bar{J}\rangle)\right.$ be $\left\{\langle i, x\rangle \mid i \in \operatorname{dom}(\mathbf{J}) \cup \operatorname{dom}(\bar{J})\right.$ and $\left.x \in \mathbf{J}[i] \cup J_{i}\right\}$. Choose $\mathrm{o}^{\mathbf{J}}\langle\langle\bar{J}\rangle$ to be the function satisfying the following demands:

- ${ }^{\mathbf{J}} \subseteq \mathrm{O}^{\mathbf{J}}\langle\bar{J}\rangle$.

- The restriction of o ${ }^{\mathbf{J}}\left\langle\langle\bar{J}\rangle\right.$ to the set $\left\{\langle i, x\rangle \mid i \in \operatorname{dom}\left(p^{*}\right) \cup \operatorname{dom}(\widetilde{\mathbf{T}})\right.$ and $\left.x \in I_{i}^{p^{*}} \cup I_{i}^{\widetilde{\mathbf{T}}}\right\}$ is identity.

- Suppose $i \in \operatorname{dom}(\bar{J}) \backslash\left(\operatorname{dom}(\mathbf{J}) \cup \operatorname{dom}\left(p^{*}\right) \cup \operatorname{dom}(\widetilde{\mathbf{T}})\right)$. Then set

$$
j=\min \left(\operatorname{dom}(\bar{K}) \backslash\left(\theta \cup \operatorname{dom}\left(p^{*}\right) \cup \operatorname{dom}(\widetilde{\mathbf{T}}) \cup \mathrm{o}_{1}^{\mathbf{J}}[\operatorname{dom}(\mathbf{J})]\right)\right),
$$

where $\mathrm{o}_{1}^{\mathbf{J}}$ is the projection map of $\mathrm{o}^{\mathbf{J}}$ to the first coordinate (which is well defined, see the demands below). Note that $K_{j}=I_{\text {lex }}$. If $i \geq \theta$ $\left(J_{i} \subseteq I_{\text {lex }}\right)$, define for all $\tau \in J_{i}$,

$$
\mathrm{o}^{\mathbf{J}}\langle\bar{J}\rangle(i, \tau)=\langle j, \tau\rangle .
$$

If $i<\theta$ (hence $J_{i}=\alpha_{i}^{*}$ ), define for all $\alpha \in J_{i}$,

$$
\mathrm{o}^{\mathbf{J}}\langle\bar{J}\rangle(i, \alpha)=\left\langle j,\left\langle\xi_{\alpha}+1\right\rangle\right\rangle
$$

where $\xi_{\alpha}=\operatorname{otp}\left(J_{i} \cap \alpha\right)$ (the order type of the set).

- Suppose $i \in \operatorname{dom}(\mathbf{J})$ and $i \geq \theta$. Then $j=\mathrm{o}_{1}^{\mathbf{J}}(i)$ is defined. For all $\tau \in J_{i}$, set

$$
\mathrm{o}^{\mathbf{J} \frown\langle\bar{J}\rangle}(i, \tau)=\langle j, \tau\rangle .
$$

- Suppose $i \in \operatorname{dom}(\mathbf{J}), i<\theta$. Let $j$ be $\mathrm{o}_{1}^{\mathbf{J}}(i), \beta^{*}$ be the smallest ordinal in $\mathbf{J}[i]$, and $\tau \in I_{\text {lex }}$ be such that $\mathrm{o}^{\mathbf{J}}\left(i, \beta^{*}\right)=\langle j, \tau\rangle$. By the definition 
$n=\operatorname{lh}(\tau)>0$ and $\tau(n-1)>0$. For all $\alpha \in J_{i} \backslash \mathbf{J}[i]$ with $\alpha<\beta^{*}$, define

$$
\mathrm{o}^{\mathbf{J}}\left\langle\langle\bar{J}\rangle(i, \alpha)=\left\langle j, \tau \uparrow(n-1) \frown\left\langle 0, \xi_{\alpha}+1\right\rangle\right\rangle,\right.
$$

where $\xi_{\alpha}=\operatorname{otp}\left(J_{i} \cap \alpha\right)$. For every $\alpha \in J_{i} \backslash\left(\mathbf{J}[i] \cup \beta^{*}\right)$, there exists a unique $\beta_{\alpha} \in \mathbf{J}[i]$ (by the assumption which makes $\mathbf{J}[i]$ closed under supremums $)$ such that $\beta_{\alpha}<\alpha<\min \left(\left(\mathbf{J}[i] \cup\left\{\alpha_{i}^{*}\right\}\right) \backslash\left(\beta_{\alpha}+1\right)\right)$. Moreover, for every such $\alpha$, there exists a unique $\tau_{\alpha} \in I_{\text {lex }}$ such that $\mathrm{o}^{\mathbf{J}}\left(i, \beta_{\alpha}\right)=\left\langle j, \tau_{\alpha}\right\rangle$. Now for all $\alpha \in J_{i} \backslash\left(\mathbf{J}[i] \cup \beta^{*}\right)$ define

$$
\mathrm{o}^{\mathbf{J}}\langle\bar{J}\rangle(i, \alpha)=\left\langle j, \tau_{\alpha} \frown\left\langle\xi_{\alpha}+1\right\rangle\right\rangle,
$$

where $\xi_{\alpha}=\operatorname{otp}\left(J_{i} \cap \alpha\right)$.

Lemma 5.11. Suppose $\mathbf{J}$ is a finite sequence of small parts of $\bar{I}$.

(a) The mapping $\mathrm{o}^{\mathbf{J}}$ determines a complete embedding $\widehat{\mathrm{o}}^{\mathbf{J}}$ from $\overline{\mathbb{P}}(\mathbf{J})$ into $\overline{\mathbb{P}}(\bar{K})$.

(b) $\widehat{\mathrm{o}}^{\mathbf{J}}\left(p^{*}\right)=p^{*}$ and $\widehat{\mathrm{J}}(\widetilde{\mathbf{T}})=\widetilde{\mathbf{T}}$.

(c) For all small parts $\bar{J}$ of $\bar{I}, \widehat{\mathrm{o}}^{\mathbf{J}} \subseteq \widehat{\mathrm{o}} \mathbf{\mathrm { J }}\langle\bar{J}\rangle$.

Proof. (a) First of all let $\mathrm{o}_{1}^{\mathbf{J}}$ and $\mathrm{o}_{2, i}^{\mathbf{J}}, i \in \operatorname{dom}(\mathbf{J})$, be the projection mappings (these exist by Definition 5.10). Define $\bar{J}^{\prime}$ to be $\left\langle J_{j}^{\prime} \mid j \in \operatorname{dom}\left(\bar{J}^{\prime}\right)\right\rangle$, where $\operatorname{dom}\left(\bar{J}^{\prime}\right)=\mathrm{o}_{1}^{\mathbf{J}}[\operatorname{dom}(\mathbf{J})]$ and for $i \in \operatorname{dom}(\mathbf{J})$ and $j=\mathrm{o}_{1}^{\mathbf{J}}(i), J_{j}^{\prime}=$ $\mathrm{o}_{2, i}^{\mathbf{J}}[\mathbf{J}[i]]$. By Definitions 5.10 and 5.3 the mapping $\mathrm{o}_{2, i}^{\mathbf{J}}$ is an order isomorphism between the linear orders $\mathbf{J}[i]$ and $J_{j}^{\prime}$ for every $i \in \operatorname{dom}(\mathbf{J})$ and $j=\mathrm{o}_{1}^{\mathbf{J}}(i)$.

Define a mapping $\widehat{\mathrm{o}}^{\mathbf{J}}$ from $\overline{\mathbb{P}}(\mathbf{J})$ onto $\overline{\mathbb{P}}\left(\bar{J}^{\prime}\right)$ by setting $\widehat{\mathrm{o}}^{\mathbf{J}}=\left\{\left\langle p, p^{\prime}\right\rangle \mid\right.$ $p \in \overline{\mathbb{P}}(\mathbf{J})\}$, where $\operatorname{dom}\left(p^{\prime}\right)=\mathrm{o}_{1}^{\mathbf{J}}[\operatorname{dom}(p)]$ for every $p \in \overline{\mathbb{P}}(\mathbf{J})$, and for every $i \in \operatorname{dom}(p)$ and $j=\mathrm{o}_{1}^{\mathbf{J}}(i)$ we have

- $p^{\prime}(j)=\left\langle\mathbf{T}^{p^{\prime}(j)}, \Gamma^{p^{\prime}(j)},\left\langle\mathbf{b}_{\eta^{\prime}}^{p^{\prime}(j)} \mid \eta^{\prime} \in \Gamma^{p^{\prime}(j)}\right\rangle, \Delta^{p^{\prime}(j)}\right\rangle ;$

- $\mathbf{T}^{p^{\prime}(j)}=\mathbf{T}^{p(i)}$;

- $\eta^{\prime}=\left\langle\mathrm{o}_{2, i}^{\mathbf{J}}\left(\xi_{l}\right) \mid l<n\right\rangle$ for every $\eta=\left\langle\xi_{l} \mid l<n\right\rangle \in \Gamma(\mathbf{J}[i])$;

- $\Gamma^{p^{\prime}(j)}=\left\{\eta^{\prime} \mid \eta \in \Gamma^{p(i)}\right\}$

- $\mathbf{b}_{\eta^{\prime}}^{p^{\prime}(j)}=\mathbf{b}_{\eta}^{p(i)}$ for every $\eta \in \Gamma(\mathbf{J}[i])$;

- $\Delta^{p^{\prime}(j)}=\left\{\left\langle\eta^{\prime}, \mathbf{t}\right\rangle \mid\langle\eta, \mathbf{t}\rangle \in \Delta^{p(i)}\right\}$.

Clearly $\widehat{\mathrm{o}}^{\mathbf{J}}$ is an isomorphism between $\overline{\mathbb{P}}(\mathbf{J})$ and $\overline{\mathbb{P}}\left(\bar{J}^{\prime}\right)$. So the claim follows from Lemma 5.1 and the fact that $J_{j}^{\prime} \subseteq K_{j}$ for every $j \in \operatorname{dom}\left(\bar{J}^{\prime}\right)$.

(b) This follows from Definition 5.10.

(c) By Definition 5.10, $\mathrm{o}^{\mathbf{J}} \subseteq \mathrm{o}^{\mathbf{J}}\langle\bar{J}\rangle$. Hence the claim follows from the definition of $\widehat{\mathrm{o}}^{\mathbf{J}}$ and $\widehat{\mathrm{o}} \sim\langle\bar{J}\rangle$. 
Lemma 5.12. Suppose $\bar{J}$ is a small part of $\bar{I}$ such that $p^{*} \in \overline{\mathbb{P}}(\bar{J})$ and $\widetilde{\mathbf{T}}$ is a $\overline{\mathbb{P}}(\bar{J})$-name. For all $\overline{\mathbb{P}}(\bar{J})$-names $\tilde{\mathbf{d}}$ and $n<\omega$, the following properties are equivalent:
(A) $\quad p^{*} \Vdash_{\overline{\mathbb{P}}(\bar{I})} \widetilde{\mathbf{d}} \in \operatorname{Br}\left(\widetilde{T}_{n}\right) ;$
(B) $\quad p^{*} \Vdash_{\overline{\mathbb{P}}(\bar{J})} \widetilde{\mathbf{d}} \in \operatorname{Br}\left(\widetilde{T}_{n}\right)$;
(C) $\quad p^{*} \Vdash_{\overline{\mathbb{P}}(\bar{K})} \widehat{\mathrm{o}}^{\bar{J}}(\widetilde{\mathbf{d}}) \in \operatorname{Br}\left(\widetilde{T}_{n}\right)$.

Proof. The equivalence between (A) and (B) follows from Lemma 5.1 together with the fact that for any $\overline{\mathbb{P}}(\bar{I})$-generic filter $G$ over $\mathrm{V}$ with $p \in G$, $H=G \cap \overline{\mathbb{P}}(\bar{J})$ is a $\overline{\mathbb{P}}(\bar{J})$-generic filter over $\mathrm{V}$ and $\mathrm{V}[H] \subseteq \mathrm{V}[G]$.

In the same manner the equivalence between $(\mathrm{B})$ and $(\mathrm{C})$ follows from Lemma 5.11(b) and Lemma 5.11(a).

Now we are ready to prove the desired conclusion. Let $\alpha^{*}$ be the supremum of $\left\{\alpha_{i}^{*} \mid i \in \theta \cap\left(\operatorname{dom}\left(p^{*}\right) \cup \operatorname{dom}(\widetilde{\mathbf{T}})\right)\right\}$. Since $\operatorname{dom}(\widetilde{\mathbf{T}})$ has cardinality $\leq \lambda$ and because of Definition 5.2(B), we have $\alpha^{*}<\theta$ and $\mu \leq \theta$, where $\mu$ denotes the successor cardinal of $\operatorname{card}\left(\left[\alpha^{*}\right]^{\lambda}\right)$.

Define $S_{0}$ to be the whole $\mu$. By our assumption (5.3) on page 210, there exists a sequence $\left\langle\widetilde{f}_{0, \alpha} \mid \alpha<\mu\right\rangle$ of nice $\overline{\mathbb{P}}(\bar{I})$-names such that for every $\alpha \in S_{0}, \widetilde{f}_{0, \alpha}$ has cardinality $\lambda$ (by $\lambda^{+}$-c.c.) and

$$
p^{*} \Vdash_{\overline{\mathbb{P}}(\bar{I})}\left\langle\widetilde{f}_{0, \alpha}\right\rangle \in \operatorname{Br}\left(\widetilde{T}_{1}\right) \text { and } \operatorname{rk}_{\widetilde{\mathbf{T}}}\left(\left\langle\tilde{f}_{\alpha, 0}\right\rangle\right) \geq \alpha .
$$

For every $\alpha<\mu$, let $\mathbf{J}^{0, \alpha}$ denote $\left\langle\bar{I}^{p^{*}}, \bar{I}^{\widetilde{\mathbf{T}}}, \bar{I}^{\widetilde{f}_{0, \alpha}}\right\rangle$ and $\widetilde{g}_{0, \alpha}$ be $\widehat{\mathrm{o}}^{0, \alpha}\left(\widetilde{f}_{0, \alpha}\right)$. By the choice of $\mu$, there exists a $\overline{\mathbb{P}}(\bar{K})$-name $\widetilde{h}_{0}$ and $S_{1} \subseteq S_{0}$ of cardinality $\mu$ such that $\widetilde{g}_{0, \alpha}=\widetilde{h}_{0}$ for every $\alpha \in S_{1}$.

By (5.4) we may choose a sequence $\left\langle\widetilde{f}_{1, \alpha} \mid \alpha \in S_{1}\right\rangle$ of $\overline{\mathbb{P}}(\bar{I})$-names such that for every $\alpha \in S_{1}, \widetilde{f}_{1, \alpha}$ has cardinality $\lambda$ and

$$
p^{*} \Vdash_{\overline{\mathbb{P}}(\bar{I})}\left\langle\widetilde{f}_{0, \alpha^{\prime}}, \widetilde{f}_{1, \alpha}\right\rangle \in \operatorname{Br}\left(\widetilde{T_{2}}\right) \text { and } \operatorname{rk}_{\widetilde{\mathbf{T}}}\left(\left\langle\widetilde{f}_{0, \alpha^{\prime}}, \widetilde{f}_{1, \alpha}\right\rangle\right) \geq \alpha,
$$

where $\alpha^{\prime}$ denotes the smallest $\beta \in S_{1}$ such that $\beta>\alpha$.

For every $\alpha \in S_{1}$, define $\mathbf{J}^{1, \alpha}$ to be $\mathbf{J}^{0, \alpha} \frown\left\langle\widetilde{I}^{\widetilde{f}_{1, \alpha}}\right\rangle$ and $\widetilde{g}_{1, \alpha}$ be $\widehat{\mathrm{o}}^{1, \alpha}\left(\widetilde{f}_{1, \alpha}\right)$. So again, there exists a $\overline{\mathbb{P}}(\bar{K})$-name $\widetilde{h}_{1}$ and $S_{2} \subseteq S_{1}$ of cardinality $\mu$ such that $\widetilde{g}_{1, \alpha}=\widetilde{h}_{1}$ for every $\alpha \in S_{2}$.

This way choose for every $n<\omega$,

- $\overline{\mathbb{P}}(\bar{I})$-names $\widetilde{f}_{n, \alpha}, \alpha \in S_{n}$,

- $\overline{\mathbb{P}}(\bar{K})$-name $\widetilde{h}_{n}$, and

- $S_{n+1} \subseteq S_{n}$

such that $\widehat{\mathrm{o}}^{\mathbf{n}, \alpha}\left(\widetilde{f}_{n, \alpha}\right)=\widetilde{h}_{n}$ for every $\alpha \in S_{n+1}$, and moreover, if $\widetilde{\mathbf{t}}_{\alpha}$, for every $\alpha \in S_{n}$, denotes a name for the sequence $\left\langle\widetilde{f}_{l, \alpha_{l}} \mid l<n+1\right\rangle$, where $\alpha_{n}=\alpha$ 
and $\alpha_{l-1}=\min S_{l} \backslash\left(\alpha_{l}+1\right)$ for every non-zero $l \leq n$, then

$p^{*} \Vdash_{\overline{\mathbb{P}}(\bar{I})}\left(\widetilde{\mathbf{t}}_{\alpha} \in \operatorname{Br}\left(\widetilde{T}_{n+1}\right)\right.$ and $\left.\operatorname{rk}_{\widetilde{\mathbf{T}}}\left(\widetilde{\mathbf{t}}_{\alpha}\right) \geq \alpha\right)$ for every $\alpha \in S_{n}$.

By Lemma 5.11, $p^{*} \Vdash_{\overline{\mathbb{P}}(\bar{K})}\left\langle\widetilde{h}_{n} \mid l<n\right\rangle \in \operatorname{Br}\left(\widetilde{T}_{n}\right)$ for every $n<\omega$. Since $\overline{\mathbb{P}}(\bar{K})$ is a complete subforcing of $\overline{\mathbb{P}}(\bar{I})$ we have $p^{*} \Vdash_{\overline{\mathbb{P}}(\bar{I})} \operatorname{rk}(\widetilde{\mathbf{T}})=\infty$.

\section{References}

[BK71] J. Barwise and K. Kunen, Hanf numbers for fragments of $L_{\infty \omega}$, Israel J. Math. 10 (1971), 306-320.

[GS] R. Grossberg and S. Shelah, On Hanf numbers of the infinitary order property, Math. Japon., submitted.

[GS86] - - - On the number of nonisomorphic models of an infinitary theory which has the infinitary order property. I, J. Symbolic Logic 51 (1986), 302-322.

[Han62] W. Hanf, Some fundamental problems concerning languages with infinitely long expressions, PhD thesis, Berkeley, 1962.

[Han64] - Incompactness in languages with infinitely long expressions, Fund. Math. 53 (1963/1964), 309-324.

[Hel64] M. Helling, Hanf numbers for some generalizations of first-order languages, Notices Amer. Math. Soc. 11 (1964), 679.

[Jec71] T. J. Jech, Trees, J. Symbolic Logic 36 (1971), 1-14.

[Kun83] K. Kunen, Set Theory, Stud. Logic Found. Math. 102, North-Holland, Amsterdam, 1983. Reprint of the 1980 original.

[LE66] E. G. K. Lopez-Escobar, On defining well-orderings, Fund. Math. 59 (1966), $13-21$.

[Mor65] M. Morley, Omitting classes of elements, in: Theory of Models (Berkeley, 1963), North-Holland, Amsterdam, 1965, 265-273.

[She90] S. Shelah, Classification Theory and the Number of Nonisomorphic Models, Stud. Logic Found. Math. 92, North-Holland, Amsterdam, 2nd ed., 1990.

[She98] - Proper and Improper Forcing, Springer, Berlin, 2nd ed., 1998.

[SV02] S. Shelah and P. Väisänen, On equivalence relations $\Sigma_{1}^{1}$-definable over $H(\kappa)$, Fund. Math. 174 (2002), 1-21.

[Sil71] J. H. Silver, Some applications of model theory in set theory, Ann. Math. Logic 3 (1971), 45-110.

Institute of Mathematics

The Hebrew University

Jerusalem, Israel

Department of Mathematics

Rutgers University

New Brunswick, NJ, U.S.A.

E-mail: shelah@math.rutgers.edu
Department of Mathematics University of Helsinki P.O. Box 4

FIN-00014, Finland E-mail: pauli.vaisanen@helsinki.fi jouko.vaananen@helsinki.fi

Received 11 March 2003; in revised form 3 June 2005 